\title{
Critical Decisions and Constitutional Rules
}

\author{
Toke S. Aidt and Francesco Giovannoni
}

May 2005

CWPE 0523

Not to be quoted without permission 


\title{
CRITICAL DECISIONS AND CONSTITUTIONAL
}

\section{RULES*}

\author{
Toke S. Aidt ${ }^{\dagger}$ \\ Faculty of Economics, University of Cambridge \\ Francesco Giovannoni $i^{\ddagger}$ \\ Department of Economics, University of Bristol
}

May 2005

\begin{abstract}
Many constitutions specify procedures that allow critical decisions to be made with a different rule than day-to-day decisions. We propose a theory of constitutional rules that explains why. The theory is based on the assumption that the type of a decision can be observed, but not verified. We characterize two classes of second-best constitutions, both with clear analogues in real world constitutions: i) incentive scheme (IS) constitutions that elicit information about the type of a decision through a costly decision rule switching procedure and ii) linking scheme (LS) constitutions that grant limited veto powers to interested parties. We explore how the relative performance of the IS and the LS constitution depends on the economic environment.

Keywords: Constitutions, social contracts, majority rules, vetoes, referenda.

JEL Classification: H10; H11.
\end{abstract}

${ }^{*}$ We would like to thank Fabrizio Adriani, Jordi Blanes i Vidal, Dhammika Dharmapala, Matthew Jackson, Matthias Messner, Miltos Makris, Giovanna Iannantuoni, seminar participants at Bristol, IGIER-Bocconi, London School of Economics, University College Dublin, University of Birmingham for helpful comments and suggestions. The usual qualifier applies.

${ }^{\dagger}$ University of Cambridge, Faculty of Economics, Austin Robinson Building, Sidgwick Ave., Cambridge CB3 9DD, UK. E-mail: toke.aidt@econ.cam.ac.uk

${ }^{\ddagger}$ Department of Economics and CMPO, University of Bristol, 8 Woodland Rd., Bristol, BS8 1TN, UK. E-mail: francesco.giovannoni@bristol.ac.uk 


\section{Introduction}

A constitution can be understood as a social contract that sets out the rules that govern the way a society makes collective decisions. The details of these contracts vary from society to society. Despite this, the world's constitutions share, in one way or the other, a common feature: procedures that allow critical decisions - decisions that, if approved, inflict large losses on some individuals - to be made using a different and stricter decision rule than that used for day-to-day decisions. The US Constitution, for example, allows the President to veto decisions made by Congress, and a veto can only be overruled by at $2 / 3$ majority of the members of Congress. Similar systems are used in many other countries (Shugart and Carey [18]). In other cases, the constitution allows citizens, through the collection of a sufficient number of signatures, to request that some policies are subjected to a referendum. The Italian and Swiss constitution provide two examples of this. A third example is the filibuster-cloture procedure in the US Senate. This procedure allows determined senators to obstruct the voting on a bill through prolonged speeches (filibuster), and a time limit on the speeches can only be imposed with the consent of 60 senators out of 100 (cloture).

The principle behind these examples is that individuals can request that critical decisions are made by a stricter decision rule, e.g., a supermajority rule or are subjected to a referendum, than the day-to-day decision rule. The challenge in implementing this principle, of course, is to identify which decisions are critical. Given that individuals who stand to lose from a particular decision would always have an incentive to claim that the decision is critical, the constitutional rules must be designed to insure that a request to transfer a decision from the domain of the day-to-day decision rule to the domain of the stricter rule is made only if the decision is truly critical. This paper proposes a theory of constitutional rules that shows how this can be achieved and argues that rules observed in actual constitutions do, in fact, achieve this objective.

We consider a society with a continuum of individuals. These individuals must choose between two alternatives $A$ and $B$. Alternative $B$ - the status quo - yields the same level of welfare to all, while alternative $A$ - a reform - creates winners and losers. The magnitude of the loss depends on the precise nature of the reform. We make a distinction between two types of reforms: critical and day-to-day reforms. Day-to-day reforms are associated with modest losses and can 
be interpreted as decisions about minor changes to the status quo. Critical reforms, on the other hand, are associated with much larger losses, e.g., because they violate certain fundamental rights. At the time when the constitutional rules are laid down, individuals do not yet know whether they will gain or lose from the reform, nor do they know the type of reform. Thus, the constitution is designed from the original position, behind a veil of ignorance. After the veil of ignorance is lifted, but before voting takes place, it becomes known if the reform is critical or not.

We begin by analyzing two benchmarks. The first benchmark characterizes the first-best constitution. The first-best is achieved by designing loss-dependent majority rules. This, however, requires that the type of reform (the loss) can be verified. This is often impossible to do and, for this reason, the constitutional rules cannot, in practice, be contingent on the utility that individuals derive from reforms. We identify this as the fundamental problem that constitutional designers must address. The second benchmark assumes that the constitutional rules are designed as if individuals do not know if a reform is critical or not before they vote on it. In this case, the constitutional choice is restricted to a single majority rule that applies to all types of reforms. We refer to this constitution as a majority rule (MR) constitution. The problem with the MR constitution is that to make it difficult to pass critical reforms, the constitutional designer makes it difficult to pass all policy reforms - something which is clearly undesirable in itself. We interpret the MR constitution as a lower bound on what constitutional designers can aspire to achieve in the realistic case where the type of reform cannot be verified objectively, but where it can be observed by individuals before voting takes place.

The fact that individuals know the type of reform before voting takes place provides constitutional designers with the opportunity to design rules that elicit this information. We consider two constitutional mechanisms that can achieve this: an incentive scheme (IS) constitution and a linking scheme (LS) constitution. Both of these embody two majority rules: a default majority rule and a stricter alternative rule that can only be applied under certain circumstances specified in the constitution.

The IS constitution allows individuals to request that the alternative rule is used, but only at a cost, $\lambda$. This cost is chosen by the constitutional designer to insure that such a request is 
only made if the reform is critical. This constitution, then, effectively elicits information about the type of reform and allows society to employ second-best, loss-dependent majority rules. The down-side is that $\lambda$ is a deadweight cost and is incurred every time the need for a switch of rules arises. Rules similar to those of the IS constitution can be found in many actual constitutions in the form of procedures that allow citizens to collect signatures to trigger referenda or citizens' initiatives.

The LS constitution takes advantage of the fact that societies have to consider many policy reforms, not just one. For each reform, the constitution allows individuals to request that the alternative rule is used instead of the default rule at no direct cost, but they can only do so a specific number of times. We interpret a request for a change of rule as a veto and the alternative rule as the majority required to overrule a veto. By rationing the number of vetoes, the constitutional designer provides incentives for revelation of the actual losses. The point is that individuals, knowing that there are more decisions to be made than vetoes allowed by the constitution, are more likely to veto a critical reform than a day-to-day reform. We argue that the LS constitution captures, in reduced form, the veto powers granted to institutions such as a president or a legislative body by actual constitutions.

The objective of both these constitutions is to establish if a reform is critical or not before it is put to a vote, but they do so in very different ways and with different costs and benefits. The IS constitution achieves full revelation, but does so at the cost $\lambda$. The LS constitution, on the other hand, is not associated with any direct costs, but it cannot guarantee full revelation: if there are too many or too few vetoes, the LS constitution sometimes allows society to make decisions with the "wrong" majority rule. The relative performance of the IS and the LS constitution and, ultimately, the choice of constitutional rules, depend on the underlying fundamentals. We undertake a detailed welfare comparison of the two types of constitutions and find that the IS constitution performs well compared to the LS constitution in situations where i) decision makers, after the veil of ignorance has been lifted, are relatively impatient; ii) critical reforms rarely appear on the agenda; and iii) support for reform is, on average, high.

The paper is organized as follows. In Section 2, we provide a brief literature review. In Section 3, we introduce our model. In Section 4, we consider the two benchmarks (the first- 
best and the MR constitution). In Section 5, we analyze the IS constitution. In Section 6, we analyze the LS constitution. In Section 7, we compare the welfare properties of the IS and the LS constitution and study the conditions under which each constitution performs better than the other. In Section 8, we provide some discussion of the results and a conclusion. The appendix at the end contains some of the proofs.

\section{Related Literature}

In recent years, there has been a renewed interest in the fundamental questions related to constitutional design. ${ }^{1}$ One strand of this literature argues that constitutions are incomplete social contracts. According to this view, constitutions cannot be designed to make policy decisions directly because constitutional designers cannot foresee all future states of nature and/or because these states of nature cannot be verified ex post. This makes it impossible or extremely costly to write a full state-contingent plan into the constitution. As a consequence, the argument goes, the role of constitutions is to define the rules that govern policy making. ${ }^{2}$ Within this framework, several papers have, under the (implicit) assumption that only voting outcomes are ex post contractible, analyzed constitutions that specify a single (majority) rule and have characterized how the optimal rule depends on the parameters of the environment. Aghion and Bolton [1] show that the optimal choice of a majority rule from behind the veil of ignorance is determined by a trade-off between two considerations. On the one hand, to limit excessive ex post redistribution (the majority expropriates the minority), a strict majority rule desirable. On the other hand, to make it harder for those who lose out (ex post vested interests) to block socially desirable reforms, a lax majority rule is desirable. ${ }^{3}$ Aghion et al. [2] focus on a rule that contains the power of political leaders. They show that the optimal degree of "insulation" measured as the share of votes needed to block legislation is determined by a trade-off between allowing the political leader enough leeway to rule and restricting the scope for misuse of power.

\footnotetext{
${ }^{1}$ The classical work in the area is Buchanan and Tullock [6]. See also Mueller [16].

${ }^{2}$ Dixit [8] presents an overview of this approach. See Laffont [13] for an exposition of the alternative view that considers constitutions as complete contracts and argues that the need for constitutions arises from information asymmetries. For a theoretical defence of the incomplete contract approach, see Hart and Moore [11].

${ }^{3}$ This approach has been further developed by Gersbach [9]. Maggi and Morelli [14] consider a similar problem in the context of international organizations.
} 
We share with these papers the incomplete contracts approach and the assumption that constitutional choices are made from behind the veil of ignorance, yet our goals are different. The existing literature focuses primarily on the problem of (excessive) ex post redistribution, and, in doing so, it makes the simplifying assumption that the cost (and benefit) of reform is certain and that all reforms are identical. We, in contrast, argue that uncertainty about the type of reforms is an important consideration in constitutional design and that societies have to take into account the fact that some reforms - those which are critical - inflict much larger costs on those who lose than day-to-day reforms. The constitutional problem, therefore, becomes one of designing rules that effectively separate critical from non-critical reforms and allow different types of reforms to be made with different rules. While the previous literature could represent constitutions as a single rule and study how this particular rule varies with changes in the underlying fundamentals, we must, as a consequence of this, deal with the choice between packages of rules and study how this choice varies with changes in the fundamentals. ${ }^{4}$ To highlight this departure from the existing literature, we do not allow for ex post redistribution. This, however, is a simplifying assumption without qualitative implications for our results: as we discuss in more detail in section 4.1, our analysis can at the cost of considerable extra complexity be extended to allow for ex post redistribution. In other words, the issue of how constitutional designers deal with situations with ex post redistribution and the issue of how they deal with situations with uncertainty about the welfare consequences of reform are in a sense orthogonal and can be analyzed separately.

Another strand of the literature has argued that constitutions are not written behind the veil of ignorance but by individuals who know their position in society. ${ }^{5}$ Messner and Polborn [15], for example, study a situation where the decision rule used to govern future decisions is itself decided by the simple majority rule. They show how supermajority rules emerge in an overlapping generations framework where the young can decide on the size of the majority that is going to be used to make decisions when they become old. Most public policies introduce immediate costs while benefits arrive later. Older voters, therefore, suffer more from reform

\footnotetext{
${ }^{4}$ Aghion and Bolton [1] take steps in this direction by analyzing when it would be desirable to introduction various minority protection rules.

${ }^{5}$ See Voigt [20] for a survey of positive constitutional economics.
} 
than young voters and this provides an incentive for young voters to choose a supermajority rule. Barbera and Jackson [5] study the broader question of when voting rules are self-sustaining and, therefore, likely to endure. They show that constitutions with two decision rules are often more stable then constitutions with a single rule. Finally, Ticchi and Vindigni [19] analyze how economic factors (and in particular redistributive concerns) influence the choice of a majoritarian versus a consensual constitution.

We have chosen to model constitutional design behind a veil of ignorance for a variety of reasons. Firstly, this assumption maps nicely with the notion that constitutional designers are "founding fathers". If the individuals who design constitutions care sufficiently about the future, they will be careful not to design a constitution that makes it either too easy or too difficult to pass reforms because while they might, for example, gain from today's reforms, they might lose from tomorrow's reforms. Secondly, any truly positive theory of constitutional design relies heavily on the details of the assumed constitutional bargaining process. In contrast, we obtain results that do not rely on anything else but the veil of ignorance assumption. Thirdly, our approach provides a benchmark against which actual outcomes might be measured and, as we shall discuss in detail below, the constitutional rules that arise from our model have clear counterparts in real world constitutions.

Our analysis is also related to a recent literature on incentive constraints and decision linking, and in our analysis of linking scheme constitutions, we draw on the framework developed by Jackson and Sonnenschein [12]. They demonstrate how a principal, by linking decisions, can elicit the agents' private information. We argue that such schemes play an important role in constitutional design.

\section{The Basic Setup}

We consider a society that is populated by a continuum of individuals of mass one, indexed by $i$. Individuals must choose between two policies, $x \in\{A, B\}$. Policy $B$ is the status quo and yields zero utility to all. Policy $A$ is an alternative to the status quo and should be interpreted as a reform, as in Aghion et al. [2]. Individuals are partitioned into two factions. Individuals in faction $W$ - the winners - prefer $A$ to $B$ and gain utility $w>0$ if the reform is chosen. We assume 
that for each individual, Nature determines independently and with probability $p$ whether she is a winner or not. Individuals in faction $W^{C}$ - the losers - prefer $B$ to $A$.

How large a loss the losers experience if policy $A$ is chosen depends on the type of reform. The type of reform is determined by the realization of the random variable $c \in\{\underline{c}, \bar{c}\}$ where $\operatorname{Pr}[c=\underline{c}]=\eta$ and $\operatorname{Pr}[c=\bar{c}]=1-\eta$. Reforms of type $\underline{c}$ are associated with small losses $(y)$ while reforms of type $\bar{c}$ are associated with large losses $(z)$. We interpret reforms of type $\underline{c}$ as non-critical, day-to-day reforms that inflict only modest losses, while reforms of type $\bar{c}$ are critical reforms that, e.g., because they violate some basic rights, inflict much larger losses. Thus, the case we have in mind is one in which $z$ is substantially larger than $y$. The type of reform cannot be known until after $c$ has been realized. We can now write the utility function of individual $i$ as follows

$$
u_{i}(x, c)=\left\{\begin{array}{ccc}
w & \text { if } & x=A \text { and } i \in W \\
-y & \text { if } & x=A, i \in W^{C} \text { and } c=\underline{c} \\
-z & \text { if } & x=A, i \in W^{C} \text { and } c=\bar{c} \\
0 & \text { if } & x=B
\end{array}\right.
$$

where $w>0$ and $z>y>0$. Each faction has a representative with the same policy preferences as the individuals in that faction. These may be called upon by the constitution to make decisions on behalf of their faction. The representatives can be interpreted as political parties, but other interpretations are possible.

We define a constitution as a mapping from i) the set of strategies of individuals and their representatives and ii) those features of the environment that are verifiable into the set $\{A, B\}$. The timing of events is summarized by the following time line:

1. From behind the veil of ignorance, one of the representatives (the constitutional designer) designs a constitution without knowing i) which faction he represents, ii) the size of each faction, and iii) the type of policy reform. ${ }^{6}$

2. Nature selects a value $p$ from a cumulative distribution function $F$ with support on the

\footnotetext{
${ }^{6}$ It does not matter which of the two representatives designs the constitutions, so we can think of the constitutional designer as being selected at random.
} 
unit interval and strictly positive density $f$. The value of $p$ is not observed by anyone.

3. Given $p$, Nature determines which faction the constitutional designer represents. By default, the other representative represents the other faction. Whether a given representative is a winner or a loser is not verifiable. Furthermore, individuals are partitioned in the two sets $W$ and $W^{C}$. Individuals know to which set they belong, but this information is not verifiable. Finally, Nature selects $c$ which is observable, but not verifiable.

4. The constitution may call upon the representatives to take some action.

5. Individuals vote for or against policy $A$. The vote result is observed by everyone and is verifiable.

6. The policy outcome is determined according to the constitutional rules laid down in 1 .

The interpretation of the time line is the following. At the constitutional stage, the two representatives do not yet know which faction they represent, nor do they know the size of each faction or the type of policy $A$ (i.e., the realization of $c$ ). As a consequence, the constitutional rules are laid down from behind the veil of ignorance, and it does not matter which of the two representatives is chosen to design the constitution. Once the constitution has been designed in stage 1, winners and losers are determined and the representatives learn which faction they represent. This takes place in two stages. In stage 2, Nature draws a value $p$ from the cumulative distribution function $F$ with support on $[0,1]$. In stage 3 , Nature determines for each individual $i$ if $i$ is a winner $(i \in W)$ or a loser $\left(i \in W^{C}\right)$. This is done by a sequence of independent draws from a Bernoulli distribution with $p=\operatorname{Pr}(i \in W)$. Nature makes one further (independent) draw from the Bernoulli distribution to determine whether the constitutional designer represents faction $W$ (with probability $p$ ) or faction $W^{C}$ (with probability $1-p$ ). By default, the other representative represents the other faction. None of this is verifiable. With a continuum of individuals, $p$ also represents the fraction of individuals who favors policy $A$ over the status quo $B{ }^{7}$ Finally, $c$ is chosen by Nature and observed by individuals and representatives. This timing

\footnotetext{
${ }^{7}$ Since the distribution of $p$ can never be deterministic, this is an abuse of the law of large numbers. See Green [10] for details.
} 
implies that the welfare consequence of policy $A$ is known before voting takes place, but after the constitution is designed. More importantly, we assume that the type of reform cannot be verified by a court. The constitutional designer, therefore, has to find ways to circumvent the fact that the constitutional rules cannot be made directly dependent on a key determinant of the desirability of reform (the realization of $c$ ). In stage 4, the constitution may allow or require the representatives to take certain actions that may reveal information about the relevant losses and determine which constitutional rule is used to make the policy decision. In stage 5, voting takes place. Individuals vote in a state of aggregate uncertainty: they know whether they are winners or losers, but they do not know how many winners or losers there are until after the vote has taken place. We assume the number of votes in favor of policy $A$ can be verified and that voting is sincere. ${ }^{8}$ This means that $p$ becomes verifiable after voting takes place and represents the measure of votes in favor of policy $A$. Finally, given the rules laid down by the constitution, policy $x$ is determined in stage 6 . We assume that a court guarantees that the constitutional rules are enforced.

\section{Benchmark Constitutions}

We begin by considering two benchmark constitutions. The first benchmark assumes that the type of the reform $(c)$ is verifiable. In this case, the first-best is attainable and can be implemented by loss-dependent majority rules. The second benchmark ignores the fact that $c$ can, in fact, be observed, and restricts the constitutional rules to depend on the number of individuals in favor of reform $(p)$. In this case, the optimal constitutional rule is a single majority rule applied to all types of reforms.

\subsection{First-Best Constitutional Rules}

Under the assumption that losses can be verified by the courts, it is possible to write constitutions that depend directly on $c$ as well as $p$. We can define a loss-dependent constitution as follows:

\footnotetext{
${ }^{8}$ This assumption is needed only because there is a continuum of voters. As a consequence, no individual voter is ever pivotal and any voting strategy, therefore, constitutes equilibrium behavior, including non-sincere ones. If, however, the probability of being pivotal were positive, sincere voting would follow from the fact that there are only two alternatives.
} 
Definition 1 A loss-dependent $(L D)$ constitution is a pair of majority rules $(m, n)$ with $m \in$ $[0,1]$ and $n \in[0,1]$ such that $t^{9,10}$

$$
x=\left\{\begin{array}{ccc}
A & \text { iff } & ((p \geq m) \wedge(c=\underline{c})) \vee((p \geq n) \wedge(c=\bar{c})) \\
B & \text { otherwise }
\end{array}\right.
$$

The LD constitution employs two loss-dependent majority rules. Non-critical reforms can pass with a $m$-majority, while critical reforms can pass with a $n$-majority. The first-best constitutional mechanism maximizes the expected utility of a representative individual from behind the veil of ignorance. The following proposition shows that the first-best constitution is a LD constitution.

Proposition 1 The $L D$ constitution with majority rules

$$
m_{F B}^{*}=\frac{y}{w+y}<\frac{z}{w+z}=n_{F B}^{*}
$$

is the first-best constitution.

Proof. From behind the veil of ignorance, the expected utility of both representatives coincides with that of a representative individual and can be written as

$$
\eta \int_{0}^{1} \operatorname{Pr}(x=A \mid \underline{c}, p) u_{\underline{c}}(p) d F(p)+(1-\eta) \int_{0}^{1} \operatorname{Pr}(x=A \mid \bar{c}, p) u_{\bar{c}}(p) d F(p)
$$

where $\operatorname{Pr}(x=A \mid c, p)$ is the probability that policy $A$ will pass under the given constitutional mechanism, conditional on $p$ and $c$, and where

$$
\begin{aligned}
& u_{\underline{c}}(p)=p w-(1-p) y ; \\
& u_{\bar{c}}(p)=p w-(1-p) z .
\end{aligned}
$$

\footnotetext{
${ }^{9}$ Since we assume that voting is sincere, we can replace, without loss of generality, the measure of votes in favor of policy $A$ with the measure $p$ of individuals in favor of policy $A$.

${ }^{10}$ The symbol " $\wedge$ " should be read as "and" while the symbol " $\vee "$ should be read as "or".
} 
This expected utility is maximized by any constitutional mechanism that satisfies the following conditions:

$$
\begin{aligned}
& \operatorname{Pr}(x=A \mid \underline{c}, p)=\left\{\begin{array}{ccc}
1 & \text { if } & u_{\underline{c}}(p) \geq 0 \Leftrightarrow p \geq \frac{y}{w+y} \\
0 & \text { otherwise }
\end{array}\right. \\
& \operatorname{Pr}(x=A \mid \bar{c}, p)=\left\{\begin{array}{ccc}
1 & \text { if } & u_{\bar{c}}(p) \geq 0 \Leftrightarrow p \geq \frac{z}{w+z} \\
0 & \text { otherwise }
\end{array} .\right.
\end{aligned}
$$

Since we assumed that individuals vote sincerely, the LD constitution with rules $\left(m_{F B}^{*}, n_{F B}^{*}\right)$ implements this outcome

The proposition shows that the constitutional designer selects a lenient majority rule $m_{F B}^{*}$ for non-critical reforms and a strict majority rule $n_{F B}^{*}$ for critical reforms involving large losses. These rules are chosen so that the first-best is achieved. We notice that these rules are independent of the distribution function $F$. Intuitively, this is because the first-best constitution requires that reforms are ex-post efficient, a requirement which is independent of $F .^{11}$

The analysis above assumes that ex post redistribution is not possible. In situations where winners could compensate losers ex post, it would seem that the optimal constitutional rule would be unanimity regardless of the realization of $c$ : only efficient policies would achieve unanimous consent. This, however, is only true if ex post redistribution can be carried out at zero deadweight cost. If we were to extend the analysis along the lines of Aghion and Bolton [1] and, like them, assume that ex post redistribution is associated with some deadweight cost, e.g., because of the cost of collecting the transfers themselves or the costs associated with identifying precisely who is a winner and who is a loser, the first-best would still require different majority rules for different types of reforms. ${ }^{12,13}$ The intuition is simple. From behind the veil of ignorance, it is not optimal to use the unanimity rule when transfers are distortionary: the deadweight loss is too large because too many losers would have to be compensated to pass a reform. Thus, it is

\footnotetext{
${ }^{11}$ Notice that the first-best constitution does not require or allow representatives to take actions in stage 4: there is no need for this when $c$ is verifiable.

${ }^{12} \mathrm{~A}$ formal analysis is available upon request.

${ }^{13}$ This result follows almost immediately from the analysis in Aghion and Bolton [1]. The optimal majority rule described there is a function of the loss that accrues to losers if a reform passes.
} 
optimal to allow reforms to pass with less than unanimity. Importantly, how lax the majority rule should be relative to unanimity depends on the size of the loss associated with the type of reform for which it is intended.

This argument demonstrates that the question of how ex post redistribution affects the design of the optimal constitutional rules is orthogonal to the question of how constitutional designers deal with uncertainty about the costs of different types of reform. The question of ex post redistribution is the main focus of previous work on constitutional rules while the focus of this paper is on the constitutional implications of uncertainty about the cost of reform. For this reason and to keep the analysis as simple as possible, we assume for the rest of the paper that ex post redistribution to "buy" a majority (or to expropriate the resources of individuals outside the majority coalition) is not possible. This assumption does not affect the qualitative nature of our results. ${ }^{14}$

\subsection{Single Majority Rules}

Under the assumption that the constitutional rules can only depend on the number of individuals in favor of reform $(p)$, a single majority rule must be applied to all decisions whether critical or not and all information about the type of reform $(c)$ is ignored. Formally, we consider the following class of constitutions:

Definition 2 A Majority-Rule (MR) constitution is a majority rule $m \in[0,1]$ such that

$$
x=\left\{\begin{array}{ccc}
A & \text { iff } & p \geq m \\
B & & \text { otherwise }
\end{array} .\right.
$$

The next proposition shows that an appropriately chosen MR constitution is the optimal constitution within the class of constitutions that solely depend on $p$.

Proposition 2 The MR constitution with majority rule

$$
m_{M R}^{*}=\frac{z-\eta(z-y)}{w+z-\eta(z-y)}
$$

is the optimal p-dependent constitution.

\footnotetext{
${ }^{14}$ It is clear, however, that the specific values of the majority rules would be different in an extended model with distortionary ex post redistribution.
} 
Proof. Write expected utility for a representative individual from behind the veil of ignorance as

$$
\int_{0}^{1} \operatorname{Pr}(x=A \mid p)\left[\eta u_{\underline{c}}(p)+(1-\eta) u_{\bar{c}}(p)\right] d F(p)
$$

where $\operatorname{Pr}(x=A \mid p)$ is the probability that policy $A$ will pass under the given constitutional mechanism, conditional on $p$ alone. Expected utility is maximized by any constitutional mechanism that satisfies the following condition:

$$
\operatorname{Pr}(x=A \mid p)=\left\{\begin{array}{ccc}
1 & \text { if } & \eta u_{\underline{c}}(p)+(1-\eta) u_{\bar{c}}(p) \geq 0 \Leftrightarrow p \geq \frac{z-\eta(z-y)}{w+z-\eta(z-y)} \\
0 & \text { otherwise }
\end{array} .\right.
$$

Given that individuals vote sincerely, the MR constitution with $m_{M R}^{*}$ implements this outcome

The optimal MR constitution applies the same majority rule to all types of reforms. This reduces expected utility below the first-best level. The welfare loss is particularly large when $z$ is large relative to $y$. In that case, the MR constitution only allows a reform to pass if supported by a large majority $\left(m_{M R}^{*}\right.$ is increasing in $\left.z\right)$ and the adaptation of all types of reforms, critical as well as non-critical ones, becomes difficult.

Taken together the two benchmarks imply that constitutional designers should strive to use loss-dependent majority rules to avoid the welfare loss associated with a single majority rule. The problem, of course, is that loss-dependent constitutional rules are not feasible when $c$ is unverifiable: whatever the type of reform is, winners have an incentive to claim that the reform is non-critical and that the decision should be made with the lenient rule $\left(m_{F B}^{*}\right)$, while the losers have an incentive to claim that all reforms are critical. Accordingly, when the type of reform is observable, but not verifiable, the challenge for the constitutional designer is to find ways to separate critical reforms from day-to-day reforms and in that way attempt to improve on the optimal MR constitution. We characterize and compare two alternative constitutional mechanisms - an incentive scheme and a linking scheme - that in different ways address this issue. We argue that these alternatives employ constitutional rules that correspond closely to solutions found in actual constitutions and identify the circumstances under which each of the alternatives is preferred to the other and to the optimal MR constitution. 


\section{Incentive Schemes}

It is common to find in actual constitutions provisions that allow policy decisions to be transferred from one decision-making procedure to another. A classic example of this is provisions for referenda or citizens initiatives. For example, the Swiss Federal Constitution says that decisions on Federal Statues and some international treaties can be submitted to a referendum at the request of 50,000 citizens. Likewise, the Italian Constitution says that 500,000 voters can request a popular referendum to decide on a total or partial repeal of a law. Another example is obstructionism in assemblies. The most famous example of this is the filibuster-cloture mechanism in the US Senate that allows minority senators to obstruct passing of bills through prolonged speeches (filibuster). ${ }^{15}$ A time limit on these speeches can only be imposed with the consent of 60 senators out of 100 (cloture), thus effectively making 60 per cent the majority rule to pass such bills. What is important about these procedures is, firstly, that the alternative procedure, explicitly or implicitly, applies a stricter threshold for approving policy or allows previous policy decisions to be overruled, and, secondly, that a cost has to be incurred in order to trigger the change in procedures. In the two referenda examples given above, the cost is that associated with collecting the required signatures; in the filibuster-cloture example, minority senators must be willing to spend considerable time and effort speaking on the floor to avoid a vote on the bill that they want to obstruct. The fact that it is costly to transfer a policy decision from the relatively lenient day-to-day decision rule (typically the simple majority rule) to the stricter alternative rule (the majority rule plus the referendum or the cloture) has an important implication: attempts to trigger a change in procedures are more likely to happen when the reform is critical. This, then, suggests that the purpose of these rules is to elicit information about the welfare losses of policy reforms; they are simple incentive schemes for truthful revelation of information about these losses. An implication is that it may be possible to design loss-dependent constitutional rules that approximate the first-best rules.

To investigate this, we consider constitutions that prescribe rules of the following type: a default majority rule that is used to make decisions unless one of the representatives, at a cost

\footnotetext{
${ }^{15}$ Although this procedure is not written down in the US constitution, it is a constitutional rule in the sense that it is a rule that governs how decisions in the Senate are made.
} 
defined in the constitution, requests that the decision is made using a different pre-specified rule. When the cost of switching from the default to the alternative rule is chosen appropriately, information about the type of reform can be elicited truthfully, and decisions can be made using loss-dependent rules. The examples above suggest that the switching cost, in real world constitutions, is a pure deadweight cost, and we begin by studying this case. This, of course, raises the question whether information about the type of reform could not be elicited truthfully at lower social cost by allowing the switching cost to be a transfer from one faction to the other. We discuss this question in section 5.2.

\subsection{Non-Transferable Resources}

We begin with the case where the switching cost is a pure deadweight loss. The time line is as in Section 3, except that we need to specify in more detail what happens in stage 4:

4. The representative for the winners $j$ and the representative for the losers $k$ simultaneously decide how large a cost $\sigma_{j} \geq 0$ and $\sigma_{k} \geq 0$ they are willing to bear to change the default decision rule to the alternative decision rule, both laid down in stage 1.

Stage 4 describes how large a cost the representatives for the two factions are willing to bear to change the decision rule from the default to the alternative rule. We assume the representatives hold enough resources to cover the cost of changing the majority rule, if they so desire. This assumption allows us to abstract from free-rider problems, although one could argue that the costs of changing the majority rule include the costs associated with the free-rider problem itself, and implies that the resources required to trigger a switch of rules are evenly distributed across individuals. We consider the following class of constitutions:

Definition 3 An incentive scheme (IS) constitution is a triplet $(m, n, \lambda)$ such that

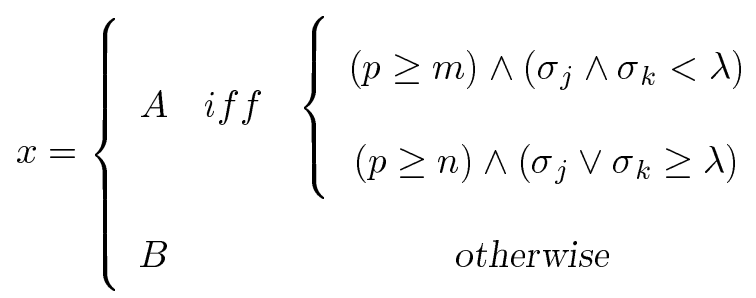


where $m \in[0,1]$ and $n \in[0,1]$ are majority rules and $\lambda \geq 0$ is the cost of changing the decision rule from $m$ to $n .^{16}$

Since the gain $(w)$ from a reform is independent on its type, only the representative for the losing faction has an incentive to spend resources triggering a switch of rules and only if the alternative rule is stricter than the default rule. ${ }^{17}$ The crucial task of the constitutional designer is to choose $\lambda, m$, and $n$ to guarantee truthful revelation of the losses, under the constraint that $\lambda$ has to be non-negative. We study subgame-perfect equilibria and assume that a representative who is indifferent between bearing and not bearing the cost $\sigma$ decides not to bear it. The next proposition characterizes the optimal IS constitution.

\section{Proposition 3 Define}

$$
\eta^{*}=\frac{(1-\psi) y(w+z)}{(z-y)((1-\psi) y+w)}
$$

where $\psi=E_{F}(p)$.

1. For $\eta>\eta^{*}$, the IS constitution with majority rules

$$
\left(m_{I S}^{*}, n_{I S}^{*}\right)=\left(\frac{y-\psi(1-\eta) y}{\eta(w+y)}, \frac{z-y(1-\psi)}{w+z}\right)
$$

and switching cost

$$
\lambda_{I S}^{*}=y\left[F\left(n_{I S}^{*}\right)-F\left(m_{I S}^{*}\right)\right]
$$

is optimal.

2. For $\eta \leq \eta^{*}$, any IS constitution that is outcome equivalent to the optimal MR constitution is an optimal IS constitution. ${ }^{18}$

3. $\eta^{*}$ is a decreasing function of $\psi, w$ and $z$ with $\lim _{z \rightarrow \infty} \eta^{*}=\frac{(1-\psi) y}{w+y(1-\psi)}, \lim _{w \rightarrow \infty} \eta^{*}=$ $(1-\psi) \frac{y}{z-y}$ and $\eta^{*}=1$ for $z=\frac{w(2-\psi)+y(1-\psi)}{w} y$.

\footnotetext{
${ }^{16}$ Technically speaking, the IS constitution consists of the whole constitutional mechanism, not just the majority rules and the cost $\lambda$. However, for simplicity, we henceforth describe each constitutional mechanism by the elements of the mechanism that the constitutional designer optimizes.

${ }^{17}$ The logic can, however, be extended to the case in which $w$ is also a function of $c$.

${ }^{18}$ Two constitutions are outcome equivalent if they induce the same probability distribution over outcomes. For example, an IS constitution with $m=m_{M R}^{*}, n=1$ and $\lambda>z\left[1-F\left(m_{M R}^{*}\right)\right]$ is outcome-equivalent to the optimal MR constitution with $m=n=m_{M R}^{*}$ and $\lambda=0$ because these constitutions share the property that only one rule is ever used and that rule is $m_{M R}^{*}$.
} 
Proof. See Appendix A.

The proposition highlights an important distinction between situations in which critical reforms are likely $\left(\eta \leq \eta^{*}\right)$ and situations in which such reforms are unlikely $\left(\eta>\eta^{*}\right)$. In the latter case, the constitutional designer selects rules that elicit the information about the losses truthfully. By defining the switching cost $\lambda$ in such a way that the representative for the losers wants to bear it if and only if policy $A$ is indeed critical, loss-dependent rules can be designed and used to make decisions. This is what makes the IS constitution attractive. The downside, of course, is that the cost $\lambda_{I S}^{*}$ is incurred whenever a critical reform appears on the agenda. Moreover, since the switching decision is made before voting reveals the value of $p$ - the fraction of winners - this cost has to be paid even if $p>n_{I S}^{*}$ (so that the reform would pass despite the switch of rule) or $p<m_{I S}^{*}$ (so that the reform would not even have passed with the default rule). Finally, the loss-dependent rules are only second-best as $m_{I S}^{*} \geq m_{F B}^{*}$ and $n_{I S}^{*} \leq n_{F B}^{*}$. To see why, we notice that the constitutional designer could, if he so desired, employ the first-best rules, but only by increasing the switching cost to $y\left[F\left(n_{F B}^{*}\right)-F\left(m_{F B}^{*}\right)\right]>\lambda_{I S}^{*}$. Part 1 of the proposition shows that it is instead optimal to distort the two majority rules in order to reduce the switching cost. Thus, the constitutional designer trades off the utility cost of reducing the difference between the two majority rules against the benefit of reducing the switching cost.

Part 2 of the proposition shows that it is not always optimal to elicit information about the losses. As noted above, the constitutional designer trades off the difference between the two lossdependent majority rules and the switching cost. When it is likely that reforms are critical and associated with large losses $\left(\eta \leq \eta^{*}\right)$ and the switching cost is incurred frequently, reducing the switching cost becomes the dominant concern. An implication, then, is that the constitutional designer wants to reduce the switching cost to zero by employing the same majority rule to all decisions. This effectively makes the IS constitution outcome equivalent to the optimal MR constitution.

Part 3 of the proposition shows that the critical value $\eta^{*}-$ that determines whether it is worthwhile to allow switching - is decreasing in $\psi, w$ and $z$. The first effect is due to the fact that from behind the veil of ignorance, an increase in $\psi$ - the expected fraction of winners makes it more likely that the constitutional designer represents the winners and, thus, does not 
have to pay the cost $\lambda$. The second effect is due to the fact that an increase in $w$ makes reforms more desirable and reduces the relative importance of the losses associated with policy $A$. The third effect is due to the fact that the benefits of using separate loss-dependent rules is larger when the two types of reforms are associated with very different losses. However, there is a limit to what an increase in $z$ can do: if $\eta$ is too small $\left(\eta<\frac{(1-\psi) y}{w+y(1-\psi)}\right)$, even very large values of $z$ (relative to $y$ ) are not sufficient to make a constitution with two separate rules optimal.

\subsection{Transferable Resources}

An important feature of the IS constitution described above is that the switching cost $\lambda$ is a deadweight cost, such as the cost of collecting signatures or the time and effort spent on setting up a filibuster, and not a transfer from one faction to the other. An alternative would be for the constitution to specify $\lambda$ in units of a resource, such as money, which is transferable between representatives, and to allow a switch to the alternative rule if one representative pays $\lambda$ to the other representative. Using transfers as a way of switching between majority rules would seem to be the efficient way to deal with the problem of eliciting the truth about the cost of reform. ${ }^{19}$

For this reason, it is important to ask why real world constitutions only allow a switch of rules at a substantial deadweight cost. We believe the main reason is that resources which can be transferred at low deadweight cost (e.g., money or promises of votes) are also resources which are likely to be unevenly distributed in society and for which it is difficult or impossible to verify differences in resource endowments objectively. As a consequence of this, the constitutional designer cannot tailor the transfer $(\lambda)$ to reflect ability to pay of the faction that seeks the switch of rules. Some faction may, therefore, occasionally not be able to pay and when this happens decisions may not be correctly separated. A trade-off between lower deadweight costs and running the risk of misallocating decisions to the "wrong" decision procedure therefore emerges. Consider, for example, an elected assembly where the constitutional rules allow for a form of obstructionism triggered by a transfer of a certain amount of money from the minority to the majority. Suppose a reform comes up for a vote. Then, if the amount of money is substantial, a minority backed by a financially weak lobby might fail to trigger a switch to the stricter rule even when the reform

\footnotetext{
${ }^{19}$ We notice that this is conceptually very different from ex post redistribution where transfers are not part of the constitution and are used as a way of overcoming a given majority rule.
} 
is critical while if the amount of money needed is small, a minority backed by a financially strong lobby would be successful in changing the rules even when the reform is not critical. As a consequence, in both cases the decision would be made by the "wrong" rule. A similar problem arises when the transfer is specified in terms of political appointments or commitments to vote in a certain way in the future.

Formally, consider the case in which $\lambda$ is freely transferable between the two factions but with two caveats. First, a fraction $\beta \in[0,1]$ of the transfer is lost: if a representative makes a payment $\lambda$ in order to switch majority rule, the other representative only gets $(1-\beta) \lambda$. The case $\beta=0$ corresponds to the case with perfect transferability, while $\beta=1$ corresponds to the case where $\lambda$ is a deadweight cost, as in the examples with signatures or the filibuster. Second, resources are unevenly distributed in society. We model this with the parameter $\gamma \in[0,1]$. It represents the probability that a representative has the resources necessary to pay the transfer $\lambda$. The critical assumption is that non-transferable resources, such as the ability to gather signatures or the time to make prolong speeches, are equally distributed in society $(\gamma=1)$ while transferable resources, such as money or cabinet posts, are not $(\gamma<1) .{ }^{20}$

We are interested in comparing the expected utility of an IS constitution with evenly distributed, but non-transferable resources $(\beta=\gamma=1)$ with an IS constitution with unevenly distributed, but transferable resources $(\gamma<1, \beta<1)$. Let $\Delta E U(\beta, \gamma)$ be the difference in expected utility between the optimal IS constitution with generic values of $\beta$ and $\gamma$ and the optimal IS constitution with $\beta=\gamma=1$. We can state the following proposition:

Proposition 4 Let $F$ be uniform and let $\eta \geq \eta^{*}$.

1. $\Delta E U(\beta, \gamma)$ is decreasing in $\beta$ and increasing in $\gamma$.

2. There exists a $\bar{z}$ such that for all $z>\bar{z}, \Delta E U(\beta, \gamma)<0$.

Proof. The optimal IS constitution with $\beta, \gamma<1$ can be derived by going through the same steps as in the proof of proposition 3. The two parts of the proposition follow by studying $\Delta E U(\beta, \gamma)$

\footnotetext{
${ }^{20}$ These are stylized assumptions. One could, for example, argue that individuals with a large endowment of money may find it easier to collect signatures. Nonetheless, the fact remains that using signatures instead of money gives the poor a better chance of using the mechanism.
} 
and its derivatives as a function of the relevant parameters ${ }^{21}$

Part 1 of the proposition shows, as one would expect, that the expected utility of the IS constitution with transferable, but unevenly distributed resources is decreasing in the deadweight cost associated with transfers and increasing when resources become more evenly distributed in society. More importantly, part 2 of the proposition shows that regardless of the values of the other parameters of the model, the IS constitution with non-transferable, but evenly distributed resources is optimal for large enough values of $z$. The reason is that the IS constitution with transferable, but unevenly distributed resources cannot guarantee that decisions are made with the correct procedure. In particular, the faith of critical reforms with high costs are occasionally decided with the default rule rather than the alternative rule and, when $z$ is large, the implied welfare loss dominates the deadweight cost associated with the IS constitution with non-transferable, but evenly distributed resources.

The practical implications of proposition 4 are clear. In reality societies occasionally have to make decisions on critical reforms that, if adopted, generate large losses ( $z$ is large). It is precisely in this situation, which we argue is the one with most empirical relevance, that the need for a package of constitutional rules that allows such decisions to be transferred to a separate (and stricter) decision procedure in a reliable way is greatest. The IS constitution with transferable, but unevenly distributed resource, cannot do this. We believe that this is one key reason why actual constitutions favor the use of what effectively corresponds to an IS constitution with nontransferable, but evenly distributed resources. There are other reasons as well, of course. For example, it is easy to see that transfers would provide perverse incentives for agenda-setting: those who hold agenda-setting powers could gain simply by threatening their opposition with high-loss policies. These incentives are absent when the switching cost is a deadweight cost. Also, transfers might be perceived as unfair and thus undermine support for the constitution. For all these reasons, we focus on the IS constitution with non-transferable resources in what follows and compare it to what seems the empirically relevant alternative: linking scheme constitutions.

\footnotetext{
${ }^{21} \mathrm{~A}$ compete proof is available upon request.
} 


\section{Linking Schemes}

Many constitutions allow some political bodies or office holders to veto decisions made by other political bodies. The leading example comes from the United States. The US constitution (article I, section 7) allows the President to veto legislation made by Congress, and the veto can only be overruled by a $2 / 3$ majority in Congress. The constitution of the Philippines provides another example. ${ }^{22}$ Here, the President can veto a complete piece of legislation - a package veto - unless the package commands a $2 / 3$ majority of all members of the Congress. However, the Philippine president is, in contrast to the US president, also allowed to veto specific items of (some) packages of legislation - a so-called line-item veto. Both of these examples are from presidential systems, but similar veto procedures can be found in some parliamentarian systems. Norway provides one example. The Norwegian constitution requires that legislation be approved separately by a majority in the "Lagting" and the "Oldelsting". This effectively gives each of the two "ting" veto power over legislation, although the veto is not absolute: if one of the two "ting" calls a "veto", the proposed legislation must be considered in the "Storting" and can only pass with a $2 / 3$ majority. ${ }^{23}$

In all these examples, a veto carries no direct cost for the body or office holder that calls it. Yet, because of damage to reputation and other indirect costs, presidents or particular parliamentary bodies are careful not to veto each and every decision that they dislike. They want to economize and only veto legislation that they really dislike. Day-to-day reforms are allowed to pass without a veto and vetoes are reserved for critical reforms that are then only implemented if they can pass the stricter test required to overrule the veto. Importantly, this type of veto procedure can be interpreted as a decision linking scheme, similar to that considered by Jackson and Sonnenschein [12], designed to elicit information about the cost of policy reforms.

To develop the idea of a linking scheme constitution, suppose that society has to make $t$ decisions, one in each of $t$ periods. The parameter $t$ should not be interpreted literally as the number of decisions that society has to make, but as a measure of the time horizon of decision makers. A society with a high value of $t$ is a society in which decision makers, once the veil

\footnotetext{
${ }^{22}$ See Article VI, Section 27 (Constitution Finder, [7])

${ }^{23}$ The "Storting" consists of all the members of the "Lagting" and the "Odelsting" in joint session.
} 
of ignorance has been lifted, are relatively patient, while a society with a low value of $t$ is one in which decision makers are more short-sighted. The linking scheme constitution prescribes three rules: a default majority rule $m_{L S}$, an alternative majority rule $n_{L S}$ and a veto procedure. The veto procedure allows the representatives (in equilibrium, the one representing the losers) to request that decisions are made with rule $n_{L S}$ instead of with rule $m_{L S}$ at no direct cost, but they can only do so a fixed number of times $s \leq t$. We interpret a switch of rules as a veto with $s$ being the maximum number of vetoes allowed by the constitution and $n_{L S}$ as the majority required to overrule the veto. Actual constitutions do not explicitly prescribe an upper bound on the number of vetoes that can be called. However, as mentioned above, reputation costs provide incentives for veto-holders to economize on vetoes and by fine tuning the legislative process, constitutional designers can, implicitly or explicitly, affect the reputation costs. For example, by restricting the veto-holder's ability to propose legislation, the incentive to economize on vetoes is enhanced simply because it is in the interest of the veto-holder to preserve a good working relationship with the body that holds the right to propose policies. Another example is the distinction between line-item and package vetoes. By allowing only package vetoes, the constitutional designer makes it harder for the veto-holder to exercise his right to veto and, again, the incentive to economize is enhanced. These examples illustrate what we attempt to capture, in reduced form, with the fixed number of vetoes. By fixing the number of vetoes appropriately and thereby linking the different decisions, the constitutional designer can provide incentives for truthful revelation of the type of reform. The point is that losers, knowing that there are more decisions to be made than vetoes, are more likely to veto a critical reform than a day-to-day reform.

We begin the analysis by modifying the time line from Section 3 by, firstly, splitting stage 3 into two sub-stages to capture the notion that decisions are made repeatedly and, secondly, by describing the details of what happens in stage 4 :

3a. Given $p$, Nature determines which faction the constitutional designer represents and individuals are partitioned in the two sets $W$ and $W^{C}$. By default, the other representative represents the other faction. Individuals know to which set they belong and the representatives know which faction they represent, but this information is not verifiable.

After stage $3 a$, there are $t>1$ policy decisions to be made. For each policy decision $v \in$ 
$\{1, \ldots, t\}$, the stages $3 \mathrm{~b}$ to 6 take place sequentially until $v=t$ :

3b. Nature makes a draw of the random variable $c$ to determine the type of reform at time $v$. All draws are independent and observable, but not verifiable.

4. If at least one veto is unused at time $v$, the two representatives $j$ and $k$ simultaneously make announcements $\chi_{j}^{v}, \chi_{k}^{v} \in\{\varnothing, V\}$. Otherwise, no announcements are made.

The timing of events implies that the winners and losers are the same each period, but that the type of the reform differs from period to period. ${ }^{24}$ We interpret an announcement $\chi^{v}=V$ at time $v$ from one of the two representatives as a veto against the reform on the agenda at time $v$ which can, then, only be implemented if it commands sufficient support to overrule the veto. An announcement $\chi^{v}=\varnothing$ corresponds to a decision not to veto. Let $s_{v}$ be the number of vetoes remaining at time $v \leq t$, i.e., the total number of vetoes $(s)$ minus the number of vetoes already used. Formally, we have

$$
s_{v}=s-\#\left\{\widehat{v}<v \mid \chi_{j}^{\widehat{v}} \vee \chi_{k}^{\widehat{v}}=V\right\}
$$

It is clear that a veto can only be called at time $v$ if $s_{v}>0$. We can now define a linking scheme constitution as follows.

Definition 4 A linking scheme $(L S)$ constitution is a triplet $\left(m_{L S}, n_{L S}, s_{L S}\right)$ such that in any period $v$ for which $s_{v}=0$

$$
x=\left\{\begin{array}{lll}
A & \text { iff } & \left(p \geq m_{L S}\right) \\
B & & \text { otherwise }
\end{array}\right.
$$

and in any period $v$ for which $s_{v} \geq 1$

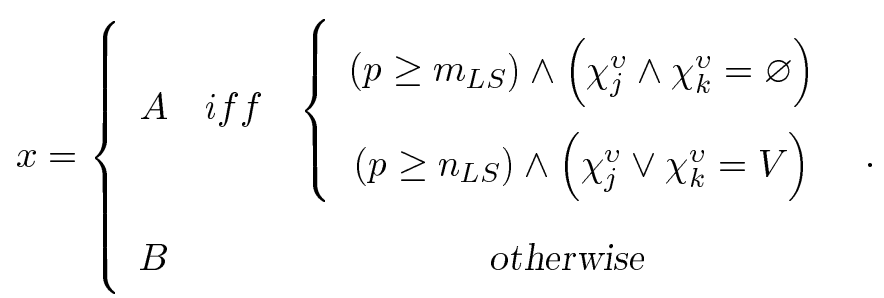

\footnotetext{
${ }^{24}$ This assumption can be relaxed. If $p$ were drawn each period and a new set of winners and losers were determined, we would get qualitatively similar results.
} 
With these definitions in place, we can characterize optimal LS constitutions. We focus on subgame-perfect equilibria and assume that a representative who is indifferent between vetoing or not does not veto.

Proposition 5 Let $E U(m, n, s)$ be the expected utility per decision associated with the $L S$ constitution $(m, n, s)$.

1. For any $1 \leq s \leq t-1$, there exist two strictly decreasing functions $m(s)$ and $n(s)$ such that $^{25}$

$$
m_{F B}^{*}<m(s)<m_{M R}^{*}<n(s)<n_{F B}^{*}
$$

Moreover, for all other majority rules $m$ and $n$

$$
E U(m(s), n(s), s)>E U(m, n, s) .
$$

2. Let

$$
s_{L S}^{*} \in \arg \max _{0 \leq s \leq t} E U(m(s), n(s), s),
$$

then $\left(m\left(s_{L S}^{*}\right), n\left(s_{L S}^{*}\right), s_{L S}^{*}\right)$ is an optimal LS constitution with $1 \leq s_{L S}^{*} \leq t-1$ for all $s_{L S}^{*}$.

3. For all $s_{L S}^{*}$

$$
\lim _{t \rightarrow \infty} E U\left(m\left(s_{L S}^{*}\right), n\left(s_{L S}^{*}\right), s_{L S}^{*}\right)=E U\left(m_{F B}^{*}, n_{F B}^{*}\right)
$$

Proof. See Appendix A.

To develop intuition for these results, it is useful to begin by assuming that $t$ is large enough to allow an accurate approximation of the binomial distribution with mean $(1-\eta) t$ and variance $\eta(1-\eta) t$ with the corresponding normal distribution. This and the fact that $t>s_{L S}^{*}>0$ imply that we can approximate the optimal number of vetoes by the solution to the following first order condition: ${ }^{26}$

$$
\Phi\left(\frac{s_{L S}^{*}-(1-\eta) t}{\sqrt{\eta(1-\eta) t}}\right) \vartheta_{1}\left(m\left(s_{L S}^{*}\right), n\left(s_{L S}^{*}\right)\right)=\left(1-\Phi\left(\frac{s_{L S}^{*}-(1-\eta) t}{\sqrt{\eta(1-\eta) t}}\right)\right) \vartheta_{2}\left(m\left(s_{L S}^{*}\right), n\left(s_{L S}^{*}\right)\right)
$$

\footnotetext{
${ }^{25}$ These functions are defined in Appendix A.

${ }^{26}$ In the continuous approximation of the expected utility function, any maximum $s_{L S}^{*}$ must satisfy this condition. Details on the derivation of this condition are available upon request.
} 
where $\Phi(\bullet)$ is the cumulative distribution function of the standard normal distribution and

$$
\begin{aligned}
& \vartheta_{1}\left(m\left(s_{L S}^{*}\right), n\left(s_{L S}^{*}\right)\right)=\int_{m\left(s_{L S}^{*}\right)}^{n\left(s_{L S}^{*}\right)}(p(w+y)-y) d F(p), \\
& \vartheta_{2}\left(m\left(s_{L S}^{*}\right), n\left(s_{L S}^{*}\right)\right)=-\int_{m\left(s_{L S}^{*}\right)}^{n\left(s_{L S}^{*}\right)}(p(w+z)-z) d F(p) .
\end{aligned}
$$

Equation (6.1) reveals an important trade-off. The left hand side represents the expected cost of allowing too many vetoes. This cost arises because of the possibility that a day-to-day reform is vetoed. Thus to be adopted it must pass the strict alternative rule when it should have been enough to pass the default rule. The per-period cost of this is $\vartheta_{1}\left(m\left(s_{L S}^{*}\right), n\left(s_{L S}^{*}\right)\right)$ and the problem arises with probability $\Phi($.$) . The right hand side represents the expected cost of allowing$ too few vetoes. This cost arises because of the possibility that a critical reform is not vetoed and thus adopted if it passes the the default rule but not necessarily the alternative rule. The per-period cost of this is given by $\vartheta_{2}\left(m\left(s_{L S}^{*}\right), n\left(s_{L S}^{*}\right)\right)$ and the problem arises with probability $1-\Phi($.$) . We, therefore, see that the optimal number of vetoes balances these two concerns.$

Interestingly, when more than one decision has to be made, part 2 of the proposition shows that it is never optimal to eliminate one of the two costs entirely by allowing either no vetoes or a veto for every decision. This implies that an optimally chosen LS constitution is always at least as good as the optimal MR constitution and strictly better for $t>1$. To see why, we notice that the MR constitution is a special case of the LS constitution with $s=0$ or $s=t$. If $s=0$, majority rule $m$ is used for all decisions because no vetoes are allowed, while if $s=t$, all decisions are vetoed by the representative of the losers and the alternative rule $n$ is used for all decisions. In both cases, the appropriate choice for the (single) majority rule is $m_{M R}^{*}$. The constitutional designer can, however, do better when more than one decision has to be made by allowing some, but not all, decisions to be vetoed. This is accomplished by adjusting the majority rules (and thus the $\operatorname{costs} \vartheta_{1}$ and $\vartheta_{2}$ ) accordingly. For example, if $s$ is small compared to $t$, the majority rule $m(s)$ is chosen to be close to $m_{M R}^{*}$ while $n(s)$ is chosen to be close to $n_{F B}^{*}$. Intuitively, this is because it is likely that the number of vetoes is too small. Thus, many decisions, regardless of their type, will be made with $m(s)$ and it is optimal to choose $m(s)$ close to $m_{M R}^{*}$. At the same time, it is highly unlikely that vetoes will be used inappropriately and this allows the constitutional designer to set $n(s)$ close to the first-best rule $n_{F B}^{*}$. Conversely, if $s$ is 
relatively close to $t$, then $m(s)$ is chosen to be close to $m_{F B}^{*}$ while $n(s)$ is chosen to be close to $m_{M R}^{*}$. In this case, most decisions are made with $n(s)$ because the representative for the losers will frequently call a veto, while $m(s)$ is likely to be used only for day-to-day decisions and so the constitutional designer sets it close to the first-best rule $m_{F B}^{*}$.

Part 3 of the proposition shows that optimally chosen LS constitutions are asymptotically first-best. ${ }^{27}$ To see the intuition, we note that the central limit theorem implies that the proportion of realizations of $c=\bar{c}$ is asymptotically distributed according to a normal distribution with mean $(1-\eta) t$ and variance $t \eta(1-\eta)$. This means that as $t$ increases, the probability of having too many or too few vetoes decreases: in the limit, this probability converges to zero. Moreover, it can be shown that as $t$ increases, the per-period expected utility associated with any optimally chosen LS constitution increases. ${ }^{28}$ An implication, then, is that optimally chosen LS constitutions, regardless of the other parameters of the model, yield higher expected utility than the optimal IS constitution, as long as $t$ is sufficiently large.

\section{Comparing Constitutional Rules}

The previous sections established some facts about the relative merits of the MR, IS and LS constitutions. Propositions 3 establishes that the IS constitution cannot improve upon the MR constitution unless critical reforms are rare $\left(\eta \geq \eta^{*}\right)$. In contrast, proposition 5 shows that for $t>1$, the LS constitution is always superior to the MR constitution. The implication is that the LS constitution is superior to both the IS and the MR constitution when critical reforms are likely $\left(\eta<\eta^{*}\right)$. Proposition 5 also establishes that the LS constitution replicates asymptotically the first-best in societies with infinitely patient decision makers $(t \rightarrow \infty)$. This, however, does not imply that the LS constitution is always superior to the IS constitution. Firstly, $t-$ a measure of how patient decision makers are - is not itself a choice variable, but a parameter of the model, and, secondly, in practice, reforms with high costs are likely to be rare events. Given that, it is

\footnotetext{
${ }^{27}$ Jackson and Sonnenshein [12] show a similar result. The main difference between the LS constitution and the mechanism they consider is that the LS constitution allows the rules $(m, n, s)$ to be determined optimally, while Jackson and Sonnenschein - using the notation of our model - set $s=(1-\eta) t$ and employ the first-best majority rules.

${ }^{28}$ Asymptotic efficiency is not sufficient to guarantee this result. A proof of the statement is available upon request.
} 
reasonable to compare optimal IS and LS constitutions for a fixed $t$. We focus on the empirically relevant case in which $z$ is large relative to $y .{ }^{29}$ To begin the analysis, define the following efficiency scores:

$$
\begin{aligned}
E S_{I S}(\eta, w, y, z, F) & =E U_{I S}(\eta, w, y, z, F)-E U_{M R}(\eta, w, y, z, F) ; \\
E S_{L S}(t, \eta, w, y, z, F) & =E U_{L S}(t, \eta, w, y, z, F)-E U_{M R}(\eta, w, y, z, F) .
\end{aligned}
$$

These efficiency scores measure the per-decision increase in expected utility, as seen from behind the veil of ignorance, of the (optimal) IS and LS constitution, respectively, relative to the (optimal) MR constitution. The efficiency scores are bounded between zero and $E U_{F B}()-.E U_{M R}($. and are functions of the key parameters of the model. Since an explicit expression for $E U_{L S}$ cannot be derived, it is not possible to compare $E S_{I S}$ and $E S_{L S}$ analytically, and we must turn to numerical simulations. ${ }^{30}$ We set $t=30$ and $w=y=1$ throughout, and study how the efficiency scores depend on $z, \eta$ and $F$.

We begin by studying $E S_{I S}$ and $E S_{L S}$ as functions of $z$ - the magnitude of the loss associated with critical reforms - and present, in Figures B.1-B.3, some simulation results for different values of $\eta .{ }^{31}$ The simulations are based on the assumption that $F$ is uniform, but similar results obtain for many other distributions. The main insight from these simulations can be summarized as follows:

Result 1 Whenever $E S_{I S}>0$,

1. $E S_{I S}$ and $E S_{L S}$ are increasing in $z$.

2. $E S_{I S}-E S_{L S}$ is increasing in $z$.

\section{INSERT FIGURES B.1-B.3 HERE}

\footnotetext{
${ }^{29}$ Proposition 4 shows that for $z$ sufficiently large, the IS constitution with non-transferable but evenly distributed resources is superior to the IS constitution with transferable but unevenly distributed resources. In all the simulations reported below, we focus on values of $z$ such that the IS constitution with $\beta=\gamma=1$ dominates any IS constitution with $\beta=\gamma<1$. For the values of $\eta$ considered, $z \geq 5 y$ is sufficient.

${ }^{30}$ The simulations are preformed with Mathematica version 5.0. The program is available upon request. In all the simulations, the optimal LS constitution is unique. Moreover, the simulations do not make use of the normal approximation of the binomial distribution.
}

${ }^{31}$ All figures are collected in Appendix B. 
Both efficiency scores are increasing in $z$. This is because it is more important to have two separate majority rules when critical reforms are much more costly than non-critical ones. More interestingly, the efficiency score of the LS constitution increases more slowly than that of the IS constitution, i.e., $E S_{I S}-E S_{L S}$ is increasing in $z$. This reflects the crucial trade-off between the two constitutions: the IS constitution is better than the LS constitution at separating reforms of different types from each other, but this advantage comes at the cost $\lambda$. The optimal LS constitution allows the "wrong" rule to be used with positive probability. The constitutional designer takes that into account by allowing $m_{L S}^{*}$ (which is used when there is no veto) as well as $n_{L S}^{*}$ (which is used when there is veto) to be increasing functions of $z^{32}$ In contrast, the optimal IS constitution never allows a decision to be made with the "wrong" majority rule. The implication, then, is that the majority rule, $m_{I S}^{*}$, used to decide the faith of non-critical reforms is independent of $z$ and only $n_{I S}^{*}$ is an increasing function of $z$. Thus, as $z$ increases, the difference between the two majority rules increases at a faster rate for the IS constitution that for the LS constitution and that accounts for the fact that $E S_{I S}-E S_{L S}$ is increasing in $z$. We note, therefore, that the IS constitution performs well relative to the LS constitution for high values of $z$.

Next, we present in Figures B.4-B.6 some simulations of $E S_{I S}$ and $E S_{L S}$ as functions of $\eta$ - the probability that reforms are non-critical - for different values of $z$. Again, we assume that $F$ is uniform, but similar results obtain for other distributions. The key insights from these simulations can be summarized as follows:

Result 2 For $E S_{I S}>0$

1. $E S_{L S}$ and $E S_{I S}$ are increasing in $\eta$ for low values of $\eta$ and decreasing in $\eta$ for high values of $\eta$.

2. $E S_{I S}-E S_{L S}$ is increasing in $\eta$.

\section{INSERT FIGURES B.4-B.6 HERE}

\footnotetext{
${ }^{32}$ The simulations show that $s_{L S}^{*}$ is also increasing in $z$. This partially offsets the impact of an increase in $z$ on both $m_{L S}^{*}$ and $n_{L S}^{*}$. However, this effect is not strong enough to change the conclusions discussed in the text.
} 
The relationship between the efficiency scores and $\eta$ is hump-shaped. This is because the advantage of having two separate majority rules rather than a single rule is largest when the uncertainty about the type of reform is high (i.e., for intermediate values of $\eta$ ). We recall from Proposition 3 that the IS constitution is outcome equivalent to the MR constitution $-E S_{I S}=0$ - for $\eta \leq \eta^{*}$. However, when $\eta>\eta^{*}$, the difference, $E S_{I S}-E S_{L S}$, is increasing in $\eta$. This is because the efficiency score of the IS constitution is affected directly by the probability of critical reforms: the lower is $\eta$ the more likely it is that the cost $\lambda$ is incurred. The efficiency score of the LS constitution is, in contrast, only affected indirectly - through the adjustment of the majority rules and the number of vetoes. Accordingly, the IS constitution performs well relative to the LS constitution for high values of $\eta$, i.e., when critical reforms are unlikely.

The efficiency scores are also affected by changes in $\psi$, the expected value of $p$. The parameter $\psi$ can be interpreted as a measure of average support for reform in a society. A society with $\psi>\frac{1}{2}$, for example, is "reform friendly" because, on average, the majority of citizens benefits from a change to the status quo, while a society with $\psi<\frac{1}{2}$ is "reform unfriendly" for the opposite reason. Moreover, in a "reform friendly" society, the constitutional designer is more optimistic about his chances of representing the winners after the constitutional rules have been drawn up and vice versa in a society that is "reform unfriendly". In Figures B.7-B.8, we presents simulations of $E S_{I S}-E S_{L S}$ as a function of $\eta$ and $z$ for different values of $\psi$. To isolate the impact of a change in the expected value of $p$ from a change in the variance, we assume that $p$ is drawn from beta distributions with the same variance as the uniform distribution, but with different expected values. ${ }^{33}$ The insights from these simulations can be summarized as follows:

Result 3 Whenever $E S_{I S}>0, E S_{I S}-E S_{L S}$ is increasing in $\psi$.

\section{INSERT FIGURES B.7-B.8 HERE}

An increase in $\psi$ - the constitutional designer becomes more optimistic about reform pos-

${ }^{33}$ Specifically, we consider the uniform distribution and beta distributions with parameters

$$
(a, b)=\left(1, \frac{1}{2}(\sqrt{33}-5)\right) \text { or }\left(\frac{1}{2}(\sqrt{33}-5), 1\right)
$$

Both of these distributions have the same variance as the uniform distribution but in the first case $\psi=\frac{2}{\sqrt{33}-3} \approx 0.73$ (the optimistic case) and in the second $\psi=\frac{\sqrt{33}-5}{\sqrt{33}-3} \approx 0.27$ (the pessimistic case). 
sibilities and his chances of representing the winners - improves the efficiency score of the IS constitution relative to that of the LS constitution. Accordingly, the IS constitution performs well relative to the LS constitution when $\psi$ is high, i.e., in "reform friendly" societies.

The preceding analysis allows us to relate the choice of constitutional rules to the underlying environment. In particular, for the empirically relevant case where $z$ is large relative to $y$ and the need for an alternative to the MR constitution is greatest, the IS constitution performs well relative to the LS constitution in societies in which:

- decision makers have a short time horizon ( $t$ is low);

- critical reforms rarely appear on the agenda ( $\eta$ is high);

- support for reform is, on average, high $\left(\psi>\frac{1}{2}\right)$.

To give an example of how these predictions can be applied in practice, consider the data reported in Shugart and Carey [18] (Table 8.2). It shows that the president of many Latin America countries has stronger veto powers than the president of the United States or heads of state in semi-presidential systems in Europe, such as France, Portugal or Ireland. The veto powers of Latin American presidents are also strong compared to European parliamentary democracies where veto powers are often restricted to sending legislation back to the parliament (e.g., in Italy) or where one chamber has limited rights to veto decisions made by another chamber (e.g., in Germany). ${ }^{34}$ This suggests that LS constitutions are more prevalent in Latin American democracies than elsewhere. If we identify a low $\eta$ with political instability - a society is politically unstable if critical and potentially disruptive reforms often appear on the agenda - and we note that political instability is relatively high in Latin American, this example is consistent with the predictions of our model.

\footnotetext{
${ }^{34}$ The Bundesrat can veto decisions made by the Bundestag for which its consent is not required according to a complex set of rules. A veto from the Bundesrat can be overridden by a simple majority in the Bundestag if the veto itself was only supported by a simple majority in the Bundesrat, while a $2 / 3$ majority in the Bundestag is required to overrule it if the veto itself was supported by a $2 / 3$ majority in the Bundesrat. Nevertheless, these rules give the Bundesrat considerably weaker veto powers than those enjoyed by almost all Latin American presidents. See Constitution Finder [7] for more details.
} 


\section{Conclusions}

We have developed a theory of constitutional design from behind the veil of ignorance. Contrary to the recent literature on the topic, we do not focus on a single exogenously given constitutional rule. Instead, study optimal packages of constitutional rules. We consider two packages of rules - IS and LS constitutions - in detail but the general approach of the paper can be extended to explain other types of rules that are often found in real world constitutions such as bills of rights, checks and balances, emergency powers and amendment rules.

Aidt and Giovannoni [3], for example, study how constitutional designers through bills of rights can partially overcome the problem that the type of reform is unverifiable. The idea is that it is possible to verify (at a cost) if a reform belongs to broad areas of possible reforms, called a policy area. As a consequence, the constitutional rules can be policy area specific, and to the extent that policies within certain policy areas are more or less likely to be critical, this can be the optimal thing to do. For example, a bill of rights can specify that policies that violate the right to a free trial can only be passed only through some special (e.g. supermajoritarian) procedure. Similarly, Aidt and Giovannoni [4] show how amendment rules and emergency powers can arise as solution to a (different) verifiability problem. In the set up of that paper, constitutions may need to allow for a change of rules in order to avoid future constitutional crises but whether a crisis is possible or not is generally not verifiable. They show that if the probability that crises are successful is very high, then constitutional amendments constitute an optimal response while if the probability that crises are successful is not so high, then allowing for emergency powers may be a better response.

It is clear, however, that our analysis leaves many important features of the collective decision problem that societies faces in reality to future research. In particular, in our model, we ignore all the agency problems that arise when decision making power is delegated to politicians. ${ }^{35}$ In addition, our policy space is very simple: in effect we have only two alternatives chosen by Nature, which differ by the loss inflicted upon losers. This means that agenda setting is not an issue and that Condorcet cycles cannot arise. Finally, we do not tackle the issue of who

\footnotetext{
${ }^{35}$ Aghion et al. [2] take a step in this direction by allowing the optimal majority rules to respond to politicians' incentives. Similary, Persson et al. [17] analyze the role of checks and balances in solving agency problems in a representative democracy.
} 
enforces the constitution. On the other hand, all these simplifications allows us to isolate what we believe to be a fundamental constitutional problem: reforms differ in their welfare effects; these effects cannot be verified, and, therefore, the constitutional rules must be designed with this in mind. Understanding why a simple decision procedure such as the majority rule is, typically, not sufficient to enable societies to resolve this problem adequately is of theoretical as well as of practical importance.

\section{References}

[1] Aghion, P. and P. Bolton (2003) "Incomplete Social Contracts". Journal of the European Economic Association 1(1): 38-67.

[2] Aghion, P., A. Alesina and F. Trebbi (2004) "Endogenous Political Institutions". Quarterly Journal of Economics 119(2): 565-611.

[3] Aidt, T. and F. Giovannoni (2004) "Constitutional Rules". CMPO working paper 04/109.

[4] Aidt, T. and F. Giovannoni (2005) "A Theory of Amendment Rules and Emergency Powers". Mimeo.

[5] Barbera, S and M. O. Jackson (2004) "Choosing How to Choose: Self-Stable Majority Rules and Constitutions". Quarterly Journal of Economics 119(3): 1011-1048.

[6] Buchanan, J.M. and G. Tullock (1965) The Calculus of Consent: Logical Foundations of Constitutional Democracy. Ann Arbor: University of Michigan Press.

[7] Constitution Finder. Available at http://confinder.richmond.edu/. Last accessed May 19th, 2004.

[8] Dixit, A.K. (1996) The Making of Economic Policy. A Transactions-cost Politics Perspective. Munich Lectures in Economics. Cambridge, MA: MIT Press.

[9] Gersbach, H. (2002) "Democratic Mechanisms: Double Majority Rules and Flexible Agenda Costs". CESifo Working Paper Series No. 749, Munich. 
[10] Green, E.J. (1994) "Individual-Level Randomness in a Non-Atomic Population", Mimeo.

[11] Hart, O. and Moore, J. (1999) "Foundations of Incomplete Contracts". Review of Economic Studies 66: 115-138.

[12] Jackson, M. O. and Sonnenschein H. F. (2005) "Overcoming Incentive Constraints by Linking Decisions". Forthcoming, Econometrica.

[13] Laffont, J.-J. (2000) Incentives and Political Economy. Clarendon Lectures in Economics. Oxford: Oxford University Press.

[14] Maggi, G., M. Morelli. (2003) "Self-enforcing Voting in International Organizations". NBER working paper no. w10102.

[15] Messner, M. and M.K. Polborn (2004) "Voting on Majority Rules". Review of Economic Studies 71: 115-132.

[16] Mueller, D. (1996) Constitutional Democracy. Oxford University Press, Oxford and New York.

[17] Persson, T, G. Roland and G. Tabellini (1997) "Separation of Powers and Political Accountability". Quarterly Journal of Economics 112: 1163-1202.

[18] Shugart, M.S. and Carey, J.M. (1992) Presidents and Assemblies. Constitutional Design and Electoral Dynamics. Cambridge, UK: Cambridge University Press.

[19] Ticchi, D. and Vindigni, A. (2003), "Endogenous Constitutions", IIES Seminar Paper No. 726, Stockholm University.

[20] Voigt, S. (1997). "Positive constitutional economics: A Survey". Public Choice 90: 11-53. 


\section{Appendix A: Proofs}

Proof of Proposition 3. Given the assumption that citizens vote sincerely, $p$ represents the fraction of society that votes for $A$ and against $B$. This allows us to ignore the voting stage (stage $5)$ and describe the IS constitution as a mechanism $\left\langle\Sigma_{j}, \Sigma_{k}, \lambda, \mu\left(\sigma_{j}, \sigma_{k}, \lambda\right)\right\rangle$ where $\Sigma_{j}=\Sigma_{k}=\Re_{+}$ are the strategy spaces for the representatives, $\sigma_{l}$ a generic strategy for representative $l$,

$$
\mu\left(\sigma_{j}, \sigma_{k}, \lambda\right)=\left\{\begin{array}{lll}
n & \text { if } & \sigma_{j} \vee \sigma_{k} \geq \lambda \\
m & & \text { otherwise }
\end{array}\right.
$$

and $\lambda \geq 0$.

First, consider the case where the constitution sets $\lambda \geq 0$ and $n \geq m$. To determine the choice of $\sigma_{j}$ and $\sigma_{k}$ in stage 4, let w.l.o.g. $j$ be the representative for the winners and $k$ the representative for the losers. It is a dominant strategy for $j$ to play $\sigma_{j}=0$. Given that, for $k$ any $\sigma_{k}<\lambda$ is dominated by $\sigma_{k}=0$ and any $\sigma_{k}>\lambda$ is dominated by $\sigma_{k}=\lambda$. Thus, in equilibrium, $k$ either plays $\sigma_{k}=0$ or $\sigma_{k}=\lambda$. By playing $\sigma_{k}=\lambda, k$ obtains

$$
\left\{\begin{array}{l}
\int_{n}^{1}-z d F(p)-\lambda \quad \text { if } \quad c=\bar{c} \\
\int_{n}^{1}-y d F(p)-\lambda \text { if } \quad c=\underline{c}
\end{array}\right.
$$

while by playing $\sigma_{k}=0, k$ obtains

$$
\left\{\begin{array}{l}
\int_{m}^{1}-z d F(p) \quad \text { if } \quad c=\bar{c} \\
\int_{m}^{1}-y d F(p) \text { if } \quad c=\underline{c}
\end{array} .\right.
$$

We restrict attention to Nash equilibria in which $k$ plays $\sigma_{k}=0$ if indifferent between $\sigma_{k}=\lambda$ and $\sigma_{k}=0$. Give that, $k^{\prime} s$ equilibrium strategy is

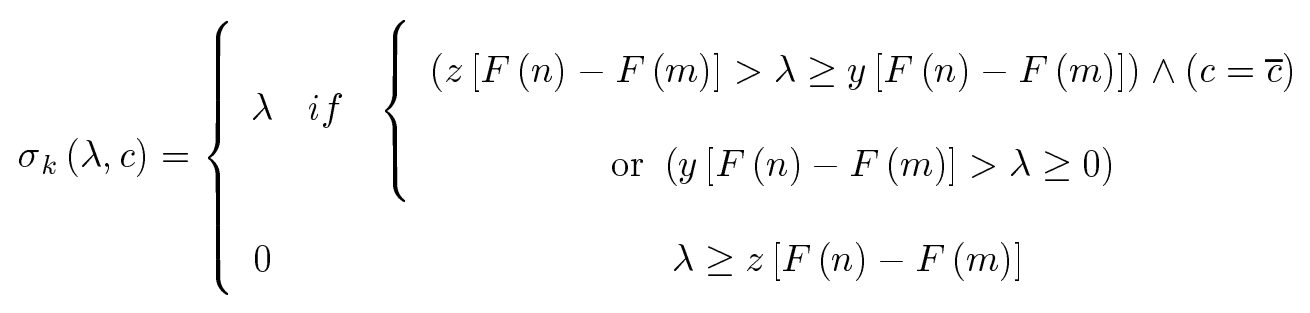

If $y[F(n)-F(m)]>\lambda \geq 0$, then $\sigma_{k}(\lambda, c)=\lambda$ for all $c$ and rule $n$ is used for all decisions, irrespectively of the type of policy $A$. Likewise, if $\lambda \geq z[F(n)-F(m)]$, then $\sigma_{k}(\lambda, c)=0$ for 
all $c$ and rule $m$ is used for all decisions. It follows from proposition 2 that it is optimal in stage 1 to set $n=m_{M R}^{*}$ in the first case and $m=m_{M R}^{*}$ in the second. The resulting IS constitutions are outcome-equivalent to the optimal MR constitutions.

Now, consider the remaining case in which $z[F(n)-F(m)]>\lambda \geq y[F(n)-F(m)]$. Since $\lambda$ is a deadweight cost, it is sufficient to consider the smallest value of $\lambda$ such that this constraint is satisfied:

$$
\lambda=y[F(n)-F(m)] .
$$

We can then write the objective function of the constitutional designer in stage 1 as

$$
\begin{gathered}
\eta \int_{m}^{1}(p(w+y)-y) d F(p)+(1-\eta) \int_{n}^{1}(p(w+z)-z) d F(p) \\
-\int_{0}^{1}(1-p) d F(p)\left((1-\eta) y \int_{m}^{n} d F(p)\right)
\end{gathered}
$$

where $\int_{0}^{1}(1-p) d F(p)=1-\psi$. Rearranging this, we obtain

$$
\begin{aligned}
& \int_{m}^{1}[\eta(p(w+y)-y)-(1-\psi)(1-\eta) y] d F(p) \\
& +\int_{n}^{1}[(1-\eta)(p(w+z)-z+(1-\psi) y)] d F(p) .
\end{aligned}
$$

This expression is maximized whenever

$$
\operatorname{Pr}(x=A \mid \underline{c}, p)=\left\{\begin{array}{ccc}
1 & \text { if } \quad \eta(p(w+y)-y)-(1-\eta)(1-\psi) y \geq 0 \Leftrightarrow p \geq \frac{y-\psi(1-\eta) y}{\eta(w+y)}=m_{I S}^{*} \\
0 & \text { otherwise }
\end{array}\right.
$$

and

$$
\operatorname{Pr}(x=A \mid \bar{c}, p)=\left\{\begin{array}{ccc}
1 & \text { if } & (1-\eta)(p(w+z)-z+(1-\psi) y) \geq 0 \Leftrightarrow p \geq \frac{z-y(1-\psi)}{w+z}=n_{I S}^{*} \\
0 & \text { otherwise }
\end{array},\right.
$$

respectively. Notice that

$$
\lambda_{I S}^{*}=y\left[F\left(n_{I S}^{*}\right)-F\left(m_{I S}^{*}\right)\right]>0 \Leftrightarrow \eta>\frac{(1-\psi) y(w+z)}{(z-y)((1-\psi) y+w)} \equiv \eta^{*}
$$

If $\eta>\eta^{*}$, the IS constitution $\left(m_{I S}^{*}, n_{I S}^{*}, \lambda_{I S}^{*}\right)$ is not outcome-equivalent to the optimal MR constitution because it allows for truthful revelation of $c$. Since the latter is feasible, this implies that $\left(m_{I S}^{*}, n_{I S}^{*}, \lambda_{I S}^{*}\right)$ is the optimal IS constitution. If $\eta<\eta^{*}$, then $n_{I S}^{*}<m_{I S}^{*}$. This violates 
the assumption that $n>m$ and it follows that no IS constitution can reveal the realization of c. Given that, any IS constitution that is outcome-equivalent to the optimal MR constitution is an optimal IS constitution. This includes $n_{I S}^{*}=m_{I S}^{*}=m_{M R}^{*}$. If $\eta=\eta^{*}$, then $\left(m_{I S}^{*}, n_{I S}^{*}, \lambda_{I S}^{*}\right)=$ $\left(m_{M R}^{*}, m_{M R}^{*}, 0\right)$.

Second, consider the case where the constitution sets $\lambda \geq 0$ and $n<m$. In this case, it is a dominant strategy for $k$ to play $\sigma_{k}=0$. The winners get $w$ whenever policy $A$ passes, independently of $c$. Given that, it is easy to show by going through the same reasoning as above there is no value of $\lambda \geq 0$ such that $j$ has a strict incentive to play $\sigma_{k}=\lambda$ in order to get $n$ instead of $m$. Thus, any IS constitution that is outcome-equivalent to the optimal MR constitution is an optimal IS constitution. Finally, the comparative statics and the limits of $\eta^{*}$ follow by inspection

Proof of Proposition 5 A LS constitution is a mechanism

$$
\left\langle\{\varnothing, V\}^{t},\{\varnothing, V\}^{t}, s,\left(\mu_{v}\left(\chi_{j}^{v}, \chi_{k}^{v}, s_{v}\right)\right)_{v=1}^{t}\right\rangle
$$

where $\{\varnothing, V\}^{t}$ are the strategy spaces for the representatives, $\chi_{l}^{v}$ a generic strategy for representative $l$ at time $v$,

$$
\mu_{v}\left(\chi_{j}^{v}, \chi_{k}^{v}, s_{v}\right)=\left\{\begin{array}{ccc}
m & \text { if } & \left(s_{v}=0\right) \vee\left(\chi_{j}^{v} \wedge \chi_{k}^{v}=\varnothing\right) \\
n & \text { if } & \left(s_{v} \geq 1\right) \wedge\left(\chi_{j}^{v} \vee \chi_{k}^{v}=V\right)
\end{array}\right.
$$

and $s_{v}$ is defined in the main text. ${ }^{36}$ Begin by considering stage 4 . We restrict attention to Nash equilibria with the property that if indifferent between vetoing or not, a representative does not veto. We assume w.l.o.g. that $k$ represents the losers and $j$ represents the winners. The equilibrium veto strategies $\left(\chi_{l}^{v}\right)_{v=1}^{t}$ for $l=j, k$ are as follows. If $n<m, k$ has no (strict) incentive to veto, whereas $j$ will veto the first $s$ decisions. If $n=m$, then neither representative has a strict incentive to veto. If $n>m, k$ has an incentive to veto, while $j$ never has a strict incentive to do so. If $c=\bar{c}$ in period $v$ and $s_{v} \geq 1$ then $k$ vetoes because in each subsequent period, expected losses $-y \eta-z(1-\eta)$ are lower than the current losses $-z$. If $c=\underline{c}$ and $s_{v}>t-v+1$, then $k$ does not veto because the loss today $-y$ is smaller than the expected loss $-y \eta-z(1-\eta)$ in

\footnotetext{
${ }^{36}$ Given the assumption that citizens vote sincerely, we do not need to include the voting stage (stage 5) in the formal description of the LS constitution.
} 
future periods. If, on the other hand, $c=\underline{c}$ and $s_{v}>t-v+1$ then there are enough vetoes left to cover all remaining periods $\left(s_{v} \geq t-v+1\right)$ and $k$ vetoes all remaining decisions. For any $(m, n, s)$, this defines the equilibrium strategies $\left(\chi_{j}^{v}\right)_{v=1}^{t}$ and $\left(\chi_{k}^{v}\right)_{v=1}^{t}$ of the two representatives and the resulting Nash equilibrium.

For $n \leq m$, the Nash equilibrium in stage 4 does not reveal any information about the realization of $c$. Given that, it is optimal for the constitutional designer in stage 1 to select a LS constitution that is outcome-equivalent to the optimal MR constitution. For $n>m$ the constitutional design problem is more involved. Begin by noting that a LS constitution with $n>m$ is associated with two types of costs, as seen from behind the veil of ignorance. One arises when a veto is called when there should not have been one, $\vartheta_{1}$. The other arises when no veto is called when there should have been one, $\vartheta_{2}$. Formally,

$$
\begin{aligned}
& \vartheta_{1}=\int_{m}^{n}(p(w+y)-y) d F(p) \\
& \vartheta_{2}=-\int_{m}^{n}(p(w+z)-z) d F(p) .
\end{aligned}
$$

Next, note that the event that exactly $s$ realizations of $\bar{c}$ will occur in $t$ within periods follows a Binomial distribution with mean $(1-\eta) t$ and variance $\eta(1-\eta) t$. Given the veto strategies described above, $\vartheta_{1}$ and $\vartheta_{2}$, we can for any $t-1 \geq s \geq 1$ write the per-period expected utility of the constitutional designer as

$$
\begin{aligned}
& \eta \int_{m}^{1}(p(w+y)-y) d F(p)+(1-\eta) \int_{n}^{1}(p(w+z)-z) d F(p) \\
& -\frac{1}{t} G(s, t) \int_{m}^{n}(p(w+y)-y) d F(p) \\
& +\frac{1}{t} H(s, t) \int_{m}^{n}(p(w+z)-z) d F(p)
\end{aligned}
$$

where

$$
\begin{aligned}
& G(s, t)=\sum_{k=0}^{s-1}(s-k)\left(\begin{array}{l}
t \\
k
\end{array}\right)(1-\eta)^{k} \eta^{t-k}=\sum_{x=0}^{s} \sum_{k=0}^{x-1}\left(\begin{array}{l}
t \\
k
\end{array}\right)(1-\eta)^{k} \eta^{t-k} ; \\
& H(s, t)=\sum_{k=s}^{t}(k-s)\left(\begin{array}{l}
t \\
k
\end{array}\right)(1-\eta)^{k} \eta^{t-k}=(1-\eta) t+\sum_{x=0}^{s} \sum_{k=0}^{x-1}\left(\begin{array}{l}
t \\
k
\end{array}\right)(1-\eta)^{k} \eta^{t-k}-s .
\end{aligned}
$$


Rearranging gives us

$$
\begin{aligned}
E U= & \int_{m}^{1}\left[\left(\eta-\frac{1}{t} G(s, t)\right)(p(w+y)-y)+\frac{1}{t} H(s, t)(p(w+z)-z)\right] d F(p) \\
& +\int_{n}^{1}\left[\left(1-\eta-\frac{1}{t} H(s, t)\right)(p(w+z)-z)+\frac{1}{t} G(s, t)(p(w+y)-y)\right] d F(p) .
\end{aligned}
$$

For $1 \leq s \leq t-1$ maximization of expected utility $(E U)$ requires that

$$
\operatorname{Pr}(x=A \mid \varnothing, p)=\left\{\begin{array}{ccc}
1 & \text { if } & \left(\eta-\frac{1}{t} G(s, t)\right)(p(w+y)-y)+\frac{1}{t} H(s, t)(p(w+z)-z) \geq 0 \\
0 & \Leftrightarrow p \geq \frac{\left(\eta-\frac{1}{t} G(s, t)\right) y+z \frac{1}{t} H(s, t)}{\left(\eta-\frac{1}{t} G(s, t)\right)(w+y)+(w+z) \frac{1}{t} H(s, t)}=m(s) \\
\text { otherwise }
\end{array}\right.
$$

and

$$
\operatorname{Pr}(x=A \mid V, p)=\left\{\begin{array}{ccc}
1 & \text { if } & \left(1-\eta-\frac{1}{t} H(s, t)\right)(p(w+z)-z)+\frac{1}{t} G(s, t)(p(w+y)-y) \geq 0 \\
0 & \Leftrightarrow p \geq \frac{\left((1-\eta)-\frac{1}{t} H(s, t)\right) z+y \frac{1}{t} G(s, t)}{\left((1-\eta)-\frac{1}{t} H(s, t)\right)(w+z)+(w+y) \frac{1}{t} G(s, t)}=n(s) \\
\text { otherwise }
\end{array}\right.
$$

For any $1 \leq s \leq t-1, m(s)$ and $n(s)$ are well-defined, strictly decreasing functions of $s$ with the property $m_{F B}^{*}<m(s)<m_{M R}^{*}<n(s)<n_{F B}^{*}$. Thus, the LS constitution $\left\{m\left(s_{L S}^{*}\right), n\left(s_{L S}^{*}\right), s_{L S}^{*}\right\}$, where

$$
s_{L S}^{*} \in \arg \max _{1 \leq s \leq t-1} E U(m(s), n(s), s)
$$

is an optimal LS constitution for $s_{L S}^{*} \in\{1, \ldots, t-1\}$. To complete the analysis, we need to check the cases $s=0$ and $s=t$ and show that neither case can be optimal. We note that the $s=0$ or $s=t$, given our veto strategies, are outcome-equivalent to the optimal MR constitution. Thus, the constitutional designer will not choose $s=0$ or $s=t$, if and only if for all $1 \leq s \leq t-1$, $E U(m(s), n(s), s)-E U\left(m_{M R}^{*}\right)<0$. This utility differential can be written as

$$
\begin{gathered}
\int_{m(s)}^{1}\left[\left(\eta-\frac{1}{t} G(s, t)\right)(p(w+y)-y)+\frac{1}{t} H(s, t)(p(w+z)-z)\right] d F(p) \\
+\int_{n(s)}^{1}\left[\left(1-\eta-\frac{1}{t} H(s, t)\right)(p(w+z)-z)+\frac{1}{t} G(s, t)(p(w+y)-y)\right] d F(p) \\
-\int_{m_{M R}^{*}}^{1}[(1-\eta)(p(w+z)-z)+\eta(p(w+y)-y)] d F(p) .
\end{gathered}
$$


Since $m(s)<m_{M R}^{*}<n(s)$ for any $1 \leq s \leq t-1$, we can rewrite this as

$$
\begin{aligned}
& \int_{m(s)}^{m_{M R}^{*}}\left[\left(\eta-\frac{1}{t} G(s, t)\right)(p(w+y)-y)+\frac{1}{t} H(s, t)(p(w+z)-z)\right] d F(p) \\
& +\int_{m_{M R}^{*}}^{n(s)}\left[\left(-\frac{1}{t} G(s, t)\right)(p(w+y)-y)+\left(\frac{1}{t} H(s, t)-(1-\eta)\right)(p(w+z)-z)\right] d F(p) .
\end{aligned}
$$

Using the definitions of $m(s)$ and $n(s)$, we conclude that both terms are positive for all $1 \leq s \leq$ $t-1$. Thus, $\left(m\left(s_{L S}^{*}\right), n\left(s_{L S}^{*}\right), s_{L S}^{*}\right)$ is an optimal LS constitution.

Finally, we need to show that the expected utility generated by an optimal LS constitution converges to the first-best as $t$ goes to infinity. To this end, notice that the expected utility associated with an optimally chosen LS constitution is bounded from below by the expected utility associated with the sub-optimal LS constitution $\left(\frac{y}{y+w}, \frac{z}{z+w},(1-\eta) t\right)$ and from above by the expected utility associated with the first-best constitution. Thus, it is sufficient to prove that the expected utility associated with $\left(\frac{y}{y+w}, \frac{z}{z+w},(1-\eta) t\right)$ converges to the first-best. Let $E U(t)$ be the per-period expected utility associated with the constitution $\left(\frac{y}{y+w}, \frac{z}{z+w},(1-\eta) t\right)$. Noting that $G((1-\eta) t, t)=H((1-\eta) t, t)$, we can write this as follows

$$
\begin{aligned}
E U(t) & =\int_{\frac{y}{y+w}}^{1}\left[\left(\eta-\frac{1}{t} G((1-\eta) t, t)\right)(p(w+y)-y)+\frac{1}{t} G((1-\eta) t, t)(p(w+z)-z)\right] d F(p) \\
& +\int_{\frac{z}{z+w}}^{1}\left[\left(1-\eta-\frac{1}{t} G((1-\eta) t, t)\right)(p(w+z)-z)+\frac{1}{t} G((1-\eta) t, t)(p(w+y)-y)\right] d F(p) \\
& =\eta \int_{\frac{y}{y+w}}^{\frac{z}{z+w}}(p(w+y)-y) d F(p)+\int_{\frac{z}{z+w}}^{1} \eta(p(w+y)-y)+(1-\eta)(p(w+z)-z) d F(p) \\
& -\frac{1}{t} G((1-\eta) t, t) \int_{\frac{y}{y+w}}^{\frac{z}{z+w}}(z-y)(1-p) d F(p) \\
& =E U\left(m_{F B}^{*}, n_{F B}^{*}\right)-\frac{1}{t} G((1-\eta) t, t) \int_{\frac{y}{y+w}}^{\frac{z}{z+w}}(z-y)(1-p) d F(p) .
\end{aligned}
$$

It follows that

$$
\lim _{t \rightarrow \infty} E U(t)=E U\left(m_{F B}^{*}, n_{F B}^{*}\right) \Leftrightarrow \lim _{t \rightarrow \infty} \frac{1}{t} G((1-\eta) t, t)=0 .
$$

For $t$ sufficiently large, we can use the Normal approximation of the Binomial distribution. Letting $\Phi(\bullet)$ be the c.d.f of the standard Normal, and $\widetilde{\Phi}(\bullet)$ that of the Normal with mean $(1-\eta) t$ and variance $t \eta(1-\eta)$, we have

$$
\frac{1}{t} G((1-\eta) t, t)=\frac{1}{t} \int_{-\infty}^{(1-\eta) t} \widetilde{\Phi}(x) d x=\frac{1}{t} \int_{-\infty}^{(1-\eta) t} \Phi\left(\frac{x-(1-\eta) t}{\sqrt{t \eta(1-\eta)}}\right) d x .
$$


Using integration by parts, what we need is

$$
\lim _{t \rightarrow \infty} \frac{1}{t} \int_{-\infty}^{(1-\eta) t} x d \Phi\left(\frac{x-(1-\eta) t}{\sqrt{t \eta(1-\eta)}}\right)=\Phi(0)(1-\eta)=\frac{1-\eta}{2}
$$

But the left-hand side above can be rewritten as

$$
\begin{gathered}
\lim _{t \rightarrow \infty} \frac{1}{2 t \sqrt{\eta(1-\eta) t}} \frac{\sqrt{2}}{\sqrt{\pi}} \times \frac{1}{2} t(1-\eta) \frac{1}{\sqrt{\frac{1}{\eta(1-\eta) t}}} \\
\times \lim _{x \rightarrow-\infty}\left(2 \eta \exp \left(-\frac{1}{2} \frac{(x-t(1-\eta))^{2}}{\eta(1-\eta) t}\right) \sqrt{\frac{1}{\eta(1-\eta) t}}-2 \eta \sqrt{\frac{1}{\eta(1-\eta) t}}+\sqrt{\pi} \sqrt{2} \operatorname{erf}\left(-\frac{1}{2} \sqrt{2} \frac{x-t(1-\eta)}{\eta(1-\eta) t \sqrt{\frac{1}{\eta(1-\eta) t}}}\right)\right)
\end{gathered}
$$

where $\operatorname{erf}(\bullet)$ represents the error function. This is equivalent to

$$
\lim _{t \rightarrow \infty}\left(\frac{1}{2 t \sqrt{\eta(1-\eta) t}} \frac{\sqrt{2}}{\sqrt{\pi}}\right) \times\left(\frac{1}{2} t(1-\eta) \frac{\sqrt{2} \sqrt{\pi}-2 \eta \sqrt{\frac{1}{\eta(1-\eta) t}}}{\sqrt{\frac{1}{\eta(1-\eta) t}}}\right)=\frac{1-\eta}{2} \frac{1}{\sqrt{\eta(1-\eta)} \sqrt{\frac{1}{\eta(1-\eta)}}}=\frac{1-\eta}{2}
$$

as desired $\square$ 


\section{Appendix B: Appendix B: Figures}

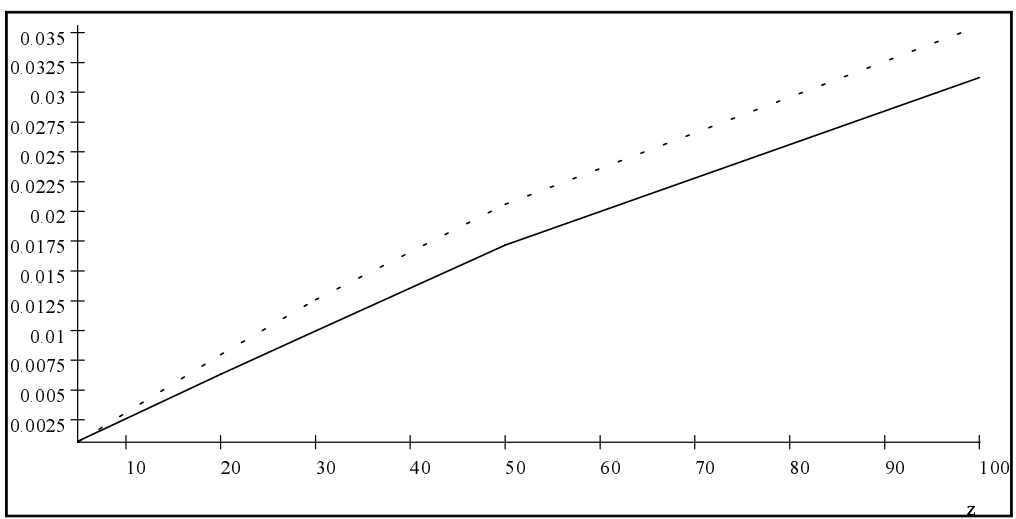

Figure B.1. Efficiency scores as a function of $z$ for $F$ uniform, $t=30, \eta=99 / 100$ and $w=y=1$. Solid line is $E S_{L S}$, dashed line is $E S_{I S}$.

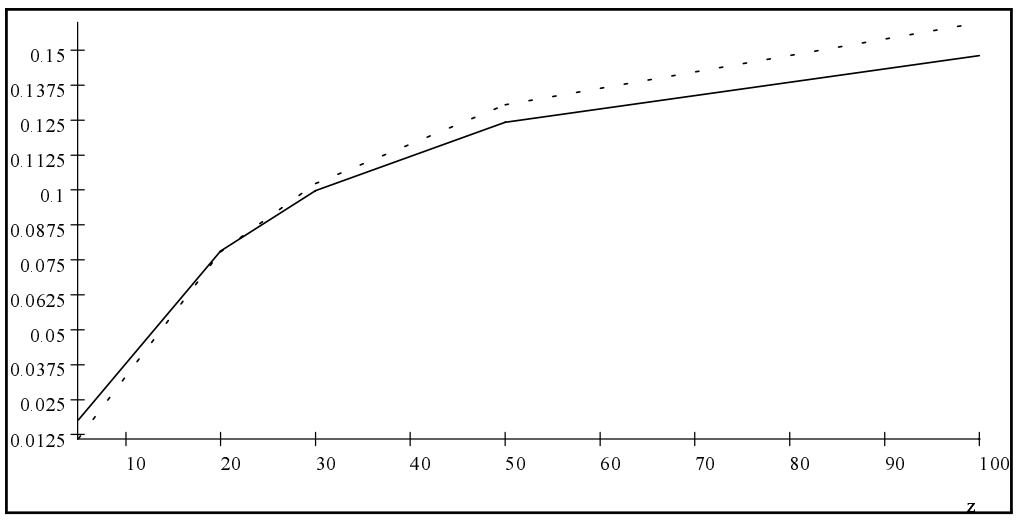

Figure B.2. Efficiency scores as a function of $z$ for $F$ uniform, $t=30, \eta=9 / 10$ and $w=y=1$. Solid line is $E S_{L S}$, dashed line is $E S_{I S}$.

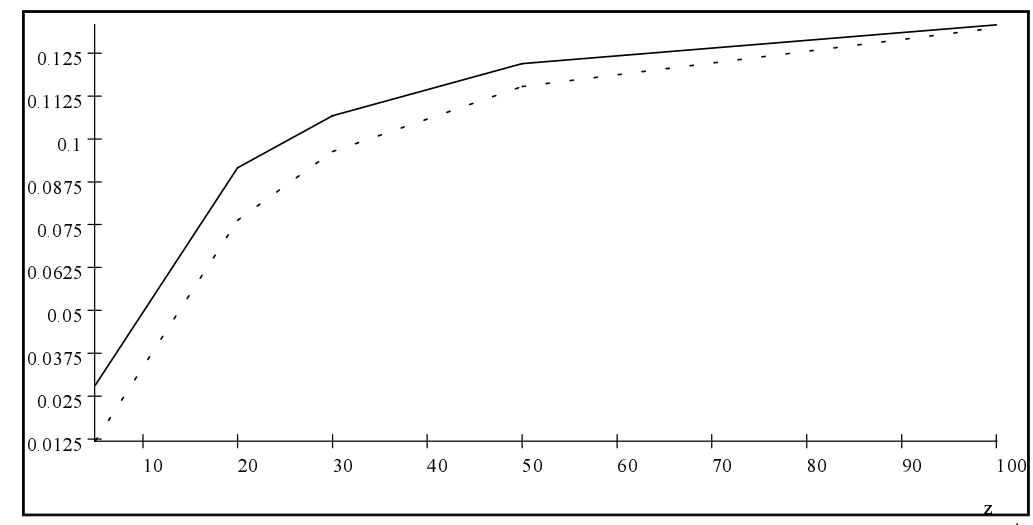

Figure B.3. Efficiency scores as a function of $z$ for $F$ uniform, $t=30, \eta=4 / 5$ and $w=y=1$. Solid line is $E S_{L S}$, dashed line is $E S_{I S}$. 


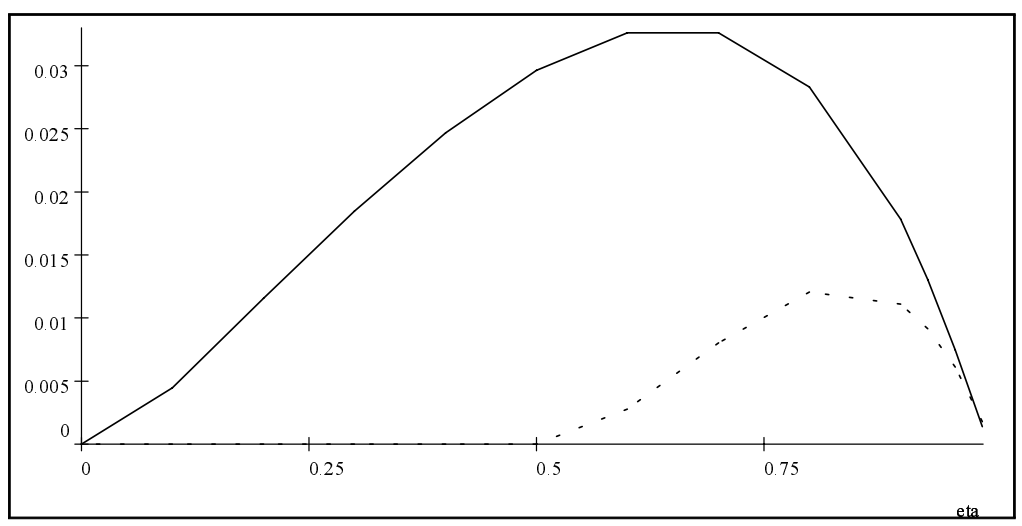

Figure B.4. Efficiency scores as a function of $\eta$ for $F$ uniform, $t=30, z=5$ and $w=y=1$. Solid line is $E S_{L S}$, dashed line is $E S_{I S}$.

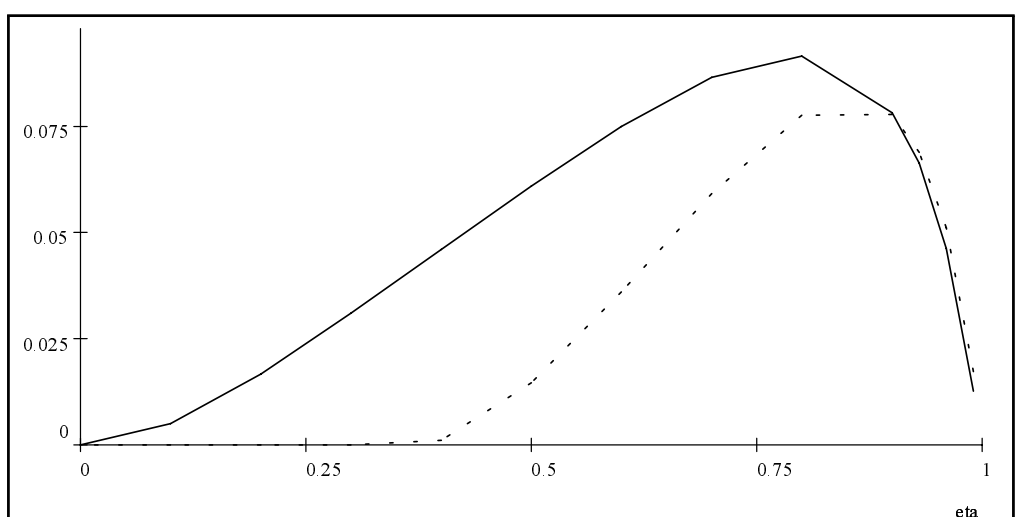

Figure B.5. Efficiency scores as a function of $\eta$ for $F$ uniform, $t=30, z=20$ and $w=y=1$. Solid line is $E S_{L S}$, dashed line is $E S_{I S}$.

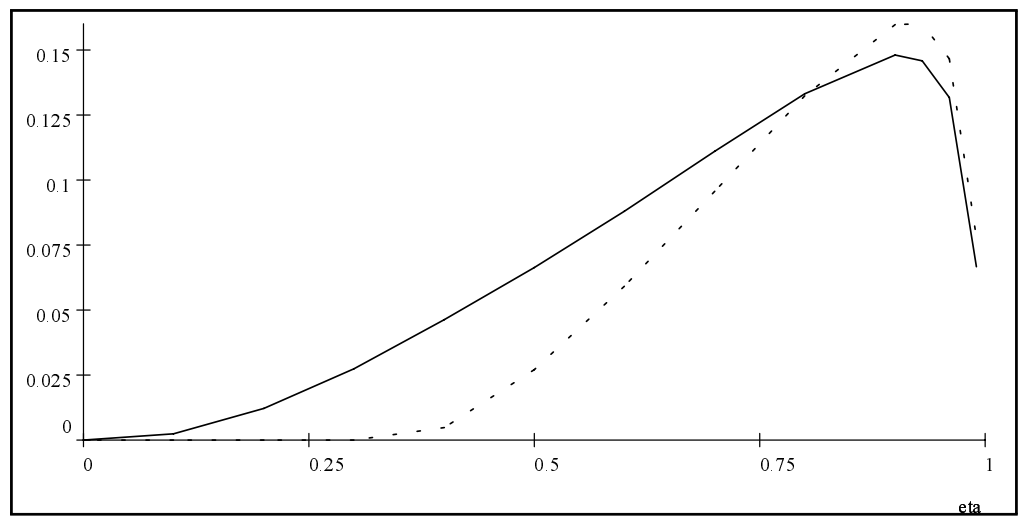

Figure B.6. Efficiency scores as a function of $\eta$ for $F$ uniform, $t=30, z=100$ and $w=y=1$. Solid line is $E S_{L S}$, dashed line is $E S_{I S}$. 


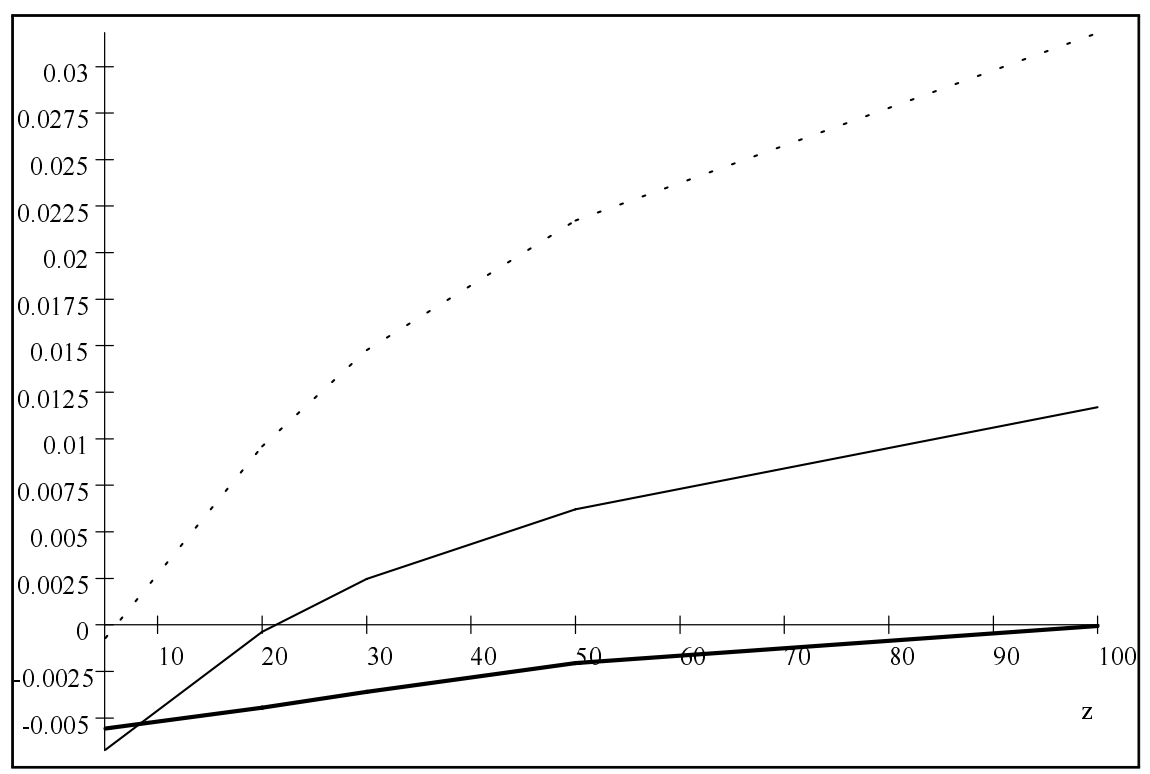

Figure B.7. $E S_{I S}-E S_{L S}$ as a function of $z$ for $t=30, \eta=9 / 10$ and $w=y=1$. Solid line $F$ is uniform, dashed line $F$ is optimistic Beta and dotted line is pessimistic Beta.

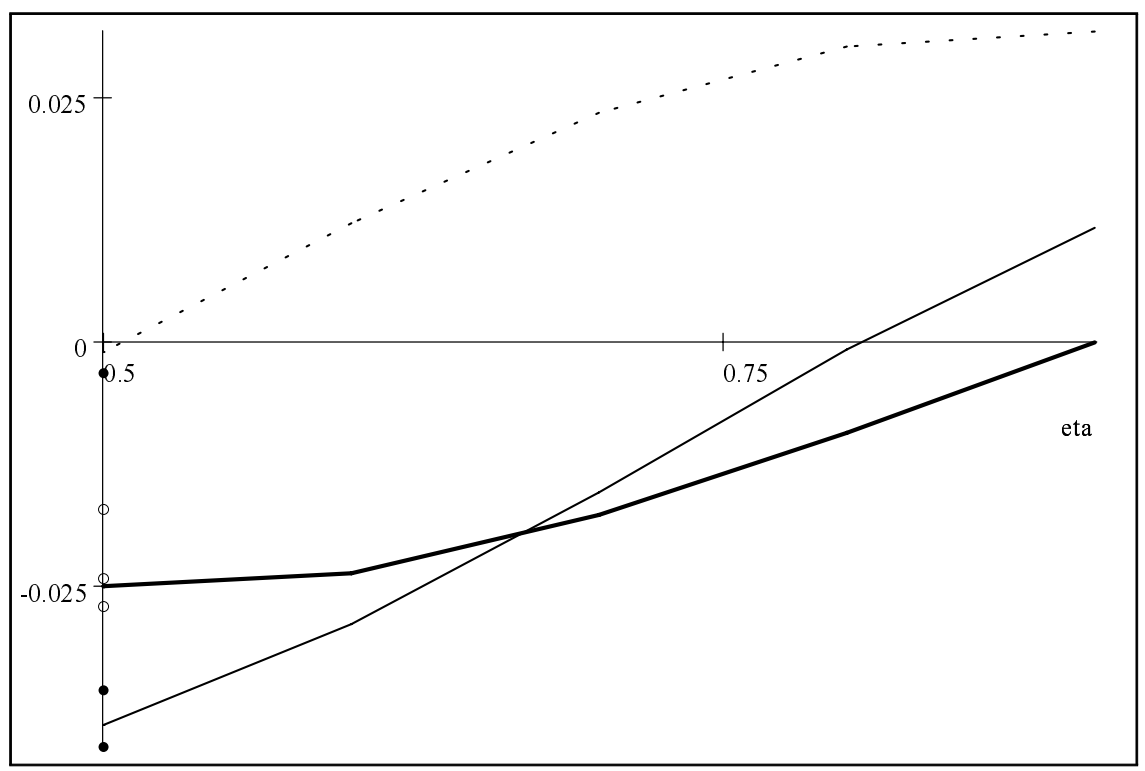

Figure B.8. $E S_{I S}-E S_{L S}$ as a function of $\eta$ for $t=30, z=100$ and $w=y=1$. Solid line $F$ is uniform, dashed line $F$ is optimistic Beta, dotted line $F$ is pessimistic Beta. 